\title{
Near-infrared surface photometry of early-type spiral galaxies
}

\author{
I. Bulge and disk decomposition ${ }^{\star}$ \\ G. Moriondo ${ }^{1}$, C. Giovanardi ${ }^{2}$, and L.K. Hunt ${ }^{3}$ \\ 1 Dipartimento di Astronomia e Scienza dello Spazio, L.E. Fermi 5, I-50125 Firenze, Italy \\ 2 Osservatorio Astrofisico di Arcetri, L.E. Fermi 5, I-50125 Firenze, Italy \\ 3 C.A.I.S.M.I., L.E. Fermi 5, I-50125 Firenze, Italy
}

Received May 22; accepted November 17, 1997

\begin{abstract}
We present near-infrared (NIR) surface photometry of a sample of 14 early-type spirals with observed rotation curves. In this first paper, we report the results of two-dimensional parametric and non-parametric decompositions to separate the bulge and disk components; the parametric bulge is modeled with a generalized exponential law of integer index $n$, and the disk with a simple exponential. We find that the derived bulge parameters, for a given galaxy, vary systematically with the bulge shape index $n$. The mean early-type bulge has a bestfit $n=2.6$, and $80 \%$ of the sample has best $n$ of 2 or 3 . Bulges are rarely spherical; the median bulge intrinsic ellipticity is 0.33 . The median early-type disk has $(J-K)_{\mathrm{d}}$ more than 0.1 mag bluer than the bulge, and a NIR disk surface brightness more than $1 \mathrm{mag} \operatorname{arcsec}^{-2}$ brighter than later-type disks. Our data are consistent with the well-established correlation of both bulge and disk surface brightness with physical scale length, and we note that the location of bulges within this projection of the fundamental plane depends on their shape index $n$. In agreement with previous work, the ratios of bulge and disk scale lengths are consistent with a constant value $r_{\mathrm{e}} / r_{\mathrm{d}}=0.3$; however, such value again depends on the bulge index $n$, implying that claims for a scale-free Hubble sequence may be premature.
\end{abstract}

Key words: galaxies: spiral — galaxies: structure infrared: galaxies

Send offprint requests to: G. Moriondo

* Based on observations taken at TIRGO (Gornergrat, Switzerland). TIRGO is operated by CAISMI-CNR, Arcetri, Firenze, Italy.

\section{Introduction}

Surface brightness distributions of external galaxies have been studied for many years but a reliable decomposition into structural components is often difficult to obtain. The reliability of models and techniques has been questioned by several authors (Kent 1986; Schombert \& Bothun 1987; Byun \& Freeman 1995), and there are several points that need further study and refinement. First, brightness distributions have often been studied as onedimensional (1-D) radial profiles extracted by averaging along elliptical annuli; such ellipses can deviate considerably from the actual isophotes, especially in highly inclined systems with a luminous bulge. The result provides therefore a distorted profile of the surface brightness along the major axis. Second, the seeing must be properly taken into account (cf., Schombert \& Bothun 1987), especially when studying the central regions where brightness gradients are highest. Third, the choice among the parametric forms of brightness distributions (exponentials, Hubble and de Vaucouleurs laws, and so on) does not depend on any physical argument, but only on their ability to fit the data. Finally the effects of internal extinction should be taken into account.

The new near-infrared (NIR) panoramic detectors have made imaging in the 1 to $2.5 \mu \mathrm{m}$ regime rather straightforward with sensitivity and accuracy comparable to those attainable at optical wavelengths. The NIR bandpasses have been advocated to be the ideal ones to study the characteristics of the galactic backbones, that is the stellar populations which make up the mass distribution in a galaxy (e.g., Rix \& Rieke 1993). The reason for this is twofold: first, the extinction is lower, by a factor of ten between the $B$ and $K$ bandpasses; and second, the emission of old stellar populations peaks in the NIR.

In this context, we have undertaken a program of NIR imaging of bright spiral galaxies with measured rotation curves. These images are used to decompose the 
luminosity distribution into bulge and disk components and then to analyze their contribution to the observed rotation curves. To overcome the methodological drawbacks mentioned above, we have developed a parametric technique to fit a two-dimensional (2-D) bulge + disk distribution to the entire image, which takes into account the effect of seeing. In addition, generalizing Kent's non-parametric approach, we have developed an iterative algorithm to deduce from the 2-D brightness distribution the contributions of bulge and disk, again taking into account the seeing. In this first paper, we report the results of the structural decomposition for a restricted sample of early-type spirals; a subsequent paper will describe the inferred mass distributions. Such systems appear well suited for studies of this kind, mainly because of the relative smoothness of their disks and their (reputedly) lower internal extinction. On the other hand, due to the low gas content, rotation in Sa's is not measured as far out as for later types, and hence they are not quite as effective for exploring dark matter properties. In several cases it has been noted that the presence of a dominant bulge might severely complicate the picture and the analysis (Kent 1988); these are galaxies with luminous bulges and slowly rising rotation curves, and some of them are also included in our sample.

\section{Observations and data reduction}

We selected 14 early-type spirals with observed rotation curves (Rubin et al. 1985; Fillmore et al. 1986), and with diameter $\leq 8$ arcmin so that they could be imaged within our field of view. The sample is listed in Table 1, objects with anomalously slowly rising rotation curves are marked with an asterisk. Morphological type and coordinates are taken from $\mathrm{NED}^{1}$. Three galaxies reside in the Virgo Cluster: NGC 4419, 4450 and 4698 (Binggeli et al. 1985). The remaining 11 are members of loose groups (Huchra \& Geller 1982; Geller \& Huchra 1983; Rubin et al. 1985; Garcia 1993). Distances were computed according to the infall model in the Third Reference Catalog of Bright Galaxies (RC3, de Vaucouleurs et al. 1991), and assuming an $H_{0}=50 \mathrm{~km} \mathrm{~s}^{-1} \mathrm{Mpc}^{-1}$. For each group or cluster its mean redshift was used; the adopted distance scale implies a Virgo distance modulus of 31.9 mag. The position angle (Col. 6) is obtained at the outer isophotes, measured East from North; the optical radius and the $B$-band magnitude (Cols. 7, 8) are from the RC3; Col. 9 reports the bands in which each galaxy has been observed.

Images were acquired at the Gornergrat Infrared Telescope (TIRGO, $1.5 \mathrm{~m}, \mathrm{f} / 20)$ in the $J(1.2 \mu \mathrm{m})$ and $K$ $(2.2 \mu \mathrm{m})$ bands, using the Arcetri NIR camera ARNICA (Lisi et al. 1996), equipped with a NICMOS 3 detector.

\footnotetext{
1 The NASA/IPAC Extragalactic Database (NED) is operated by the Jet Propulsion Laboratory, California Institute of Technology, under contract with the U.S. National Aeronautics and Space Administration.
}

The field of view of the camera is $4 \times 4$ arcmin with a plate scale of 0.97 arcsec pixel $^{-1}$. Galaxies were observed by spending half of the total integration time $(\sim 15 \mathrm{~min})$ on source, and half on the sky, alternating sky and source frames. The seeing FWHM was typically $2^{\prime \prime}$, and sky magnitudes were roughly 15.5 and $12.5 \mathrm{mag} \operatorname{arcsec}^{-2}$ in $J$ and $K$, respectively. Flat fields were obtained from sky frames; the final images are typically flat to $0.3-0.5 \%$ in the $J$ band, and to $0.1 \%$ or better in the $K$ band, giving $(1 \sigma)$ limiting magnitudes of 21.5 and $20.5 \mathrm{mag} \operatorname{arcsec}^{-2}$ in $J$ and $K$ respectively. All image reduction was performed with IRAF and the STSDAS packages ${ }^{2}$. Standard stars were selected from the UKIRT Faint Standard List (Casali \& Hawarden 1992). Zero points were derived for each night of observations after correcting for atmospheric extinction using mean TIRGO values (0.11 and 0.07 mag airmass ${ }^{-1}$ in $J$ and $K$ respectively; Hunt et al. 1987). Images were then corrected for extinction within our Galaxy and for redshift. Galactic extinction was determined according to the Burstein-Heiles values given in RC3, using the extinction curve given in Cardelli et al. (1989); K-corrections for redshift were calculated from the coefficients given by Frogel et al. (1978).

Color images were made by combining the $J$ - and $K$ band magnitude images after first registering them to a fraction of a pixel. When the seeing differed significantly between the two bands, the higher-resolution image was degraded to the level of the lower resolution one. The $K$ band images and $J-K$ color images are shown in the top panels of Fig. 1.

For five of the sample galaxies we can compare the $K$-band major-axis profiles with low-resolution $\left(28^{\prime \prime}\right.$ aperture) profiles obtained with a single-element photometer (Giovanardi \& Hunt 1996); the data are shown as filled circles in Fig. 1. As can be seen, the agreement is quite good in spite of the pointing uncertainties in the photometer profiles.

\section{Quantitative morphology}

\subsection{The parametric decomposition}

For the 2-D parametric fits, we have modeled the surface brightness distribution with a generalized exponential bulge (Sèrsic 1982; Sparks 1988):

$I_{\mathrm{b}}(x, y)=$

${ }^{2}$ IRAF is the Image Analysis and Reduction Facility made available to the astronomical community by the National Optical Astronomy Observatories, which are operated by AURA, Inc., under contract with the U.S. National Science Foundation. STSDAS is distributed by the Space Telescope Science Institute, which is operated by the Association of Universities for Research in Astronomy (AURA), Inc., under NASA contract NAS 5-26555. 
Table 1. The galaxy sample

\begin{tabular}{|c|c|c|c|c|c|c|c|c|c|c|c|c|}
\hline \multirow{2}{*}{$\begin{array}{l}\text { Name } \\
(1)\end{array}$} & \multirow{2}{*}{$\begin{array}{c}\text { Type } \\
(2)\end{array}$} & \multicolumn{3}{|c|}{ R.A. (1950) } & \multicolumn{3}{|c|}{ Dec. $(1950)$} & \multirow{2}{*}{$\begin{array}{r}\text { Distance } \\
(\mathrm{Mpc}) \\
(5)\end{array}$} & \multirow{2}{*}{$\begin{array}{r}\text { Pos. angle } \\
\text { (deg) } \\
(6)\end{array}$} & \multirow{2}{*}{$\begin{array}{c}R_{25} \\
(\operatorname{arcsec}) \\
(7)\end{array}$} & \multirow{2}{*}{$\begin{array}{c}m_{B} \\
(\mathrm{mag}) \\
(8)\end{array}$} & \multirow{2}{*}{$\begin{array}{c}\text { Band } \\
(9)\end{array}$} \\
\hline & & $\mathrm{h}$ & $\begin{array}{l}\mathrm{m} \\
(3)\end{array}$ & $\mathrm{s}$ & $\circ$ & $\begin{array}{l}\prime \\
(4)\end{array}$ & $\prime \prime$ & & & & & \\
\hline N1024 & $\left(\mathrm{R}^{\prime}\right) \mathrm{SA}(\mathrm{r}) \mathrm{ab}$ & 02 & 36 & 30.4 & 10 & 37 & 56 & 69.2 & 155 & 117 & 13.08 & $K$ \\
\hline N2639 & (R)SA(r)a:? Sy1 & 08 & 40 & 03.0 & 50 & 23 & 11 & 70.4 & 140 & 55 & 12.56 & $J, K$ \\
\hline $\mathrm{N} 2775$ & $\mathrm{SA}(\mathrm{r}) \mathrm{ab}$ & 09 & 07 & 41.0 & 07 & 14 & 35 & 27.1 & 155 & 128 & 11.03 & $J, K$ \\
\hline $\mathrm{N} 2841^{*}$ & $\mathrm{SA}(\mathrm{r}) \mathrm{b}:$ & 09 & 18 & 35.8 & 51 & 12 & 31 & 19.2 & 147 & 244 & 10.09 & $J, K$ \\
\hline N3593 & $\mathrm{SA}(\mathrm{s}) 0 / \mathrm{a}:$ & 11 & 11 & 59.2 & 13 & 05 & 28 & 17.2 & 92 & 157 & 11.86 & $J, K$ \\
\hline N3898* & $\mathrm{SA}(\mathrm{s}) \mathrm{ab}$ & 11 & 46 & 36.1 & 56 & 21 & 42 & 34.8 & 107 & 131 & 11.60 & $J, K$ \\
\hline N4378 & (R)SA(s)a & 12 & 22 & 44.8 & 05 & 12 & 06 & 51.4 & 167 & 87 & 12.63 & $J, K$ \\
\hline N4419 & $\mathrm{SB}(\mathrm{s}) \mathrm{a}$ & 12 & 24 & 24.6 & 15 & 19 & 24 & 23.9 & 133 & 99 & 12.08 & $J, K$ \\
\hline N4450 & $\mathrm{SA}(\mathrm{s}) \mathrm{ab}$ & 12 & 25 & 58.0 & 17 & 21 & 42 & 23.9 & 11 & 157 & 10.90 & $J, K$ \\
\hline N4698 & $\mathrm{SA}(\mathrm{s}) \mathrm{ab}$ & 12 & 45 & 51.3 & 08 & 45 & 35 & 23.9 & 168 & 119 & 11.46 & $J, K$ \\
\hline N4845 & $\mathrm{SA}(\mathrm{s}) \mathrm{ab}$ & 12 & 55 & 28.1 & 01 & 50 & 42 & 27.1 & 89 & 150 & 12.10 & $J, K$ \\
\hline N5879* & $\mathrm{SA}(\mathrm{rs}) \mathrm{bc}: ?$ & 15 & 08 & 27.6 & 57 & 11 & 25 & 26.2 & 2 & 125 & 12.22 & $J, K$ \\
\hline N6314 & $\mathrm{SA}(\mathrm{s}) \mathrm{a}: \mathrm{sp}$ & 17 & 10 & 33.1 & 23 & 19 & 43 & 139.2 & 177 & 43 & 13.80 & $J, K$ \\
\hline IC724 & $\mathrm{Sa}$ & 11 & 41 & 00.1 & 09 & 13 & 10 & 125.6 & 60 & 70 & 13.40 & $J, K$ \\
\hline
\end{tabular}

$$
I_{\mathrm{e}} \exp \left\{-\alpha_{n}\left[\left(\frac{1}{r_{\mathrm{e}}} \sqrt{x^{2}+\frac{y^{2}}{\left(1-\epsilon_{\mathrm{b}}\right)^{2}}}\right)^{1 / n}-1\right]\right\},
$$

plus an exponential thin disk:

$I_{\mathrm{d}}(x, y)=I_{\mathrm{d}}(0) \exp \left[-\frac{1}{r_{\mathrm{d}}} \sqrt{x^{2}+\frac{y^{2}}{\cos ^{2} i}}\right]$.

$I_{\mathrm{e}}$ and $r_{\mathrm{e}}$ are effective (half-light) values, $\epsilon_{\mathrm{b}}$ is the apparent bulge ellipticity, $\alpha_{n}$ is a constant relating the effective brightness and radius to the exponential values (see Appendix). $x$ and $y$ are in arbitrary units, with origin at the galaxy's center, and $x$ along the major axis. The bulge is assumed to be an oblate rotational ellipsoid, coaxial with the disk, and its apparent eccentricity $e_{\mathrm{b}}$ is related to the intrinsic eccentricity $e_{\mathrm{b}}^{\prime}$ by:

$e_{\mathrm{b}}=e_{\mathrm{b}}^{\prime} \sin i$.

The structural parameters have been determined by fitting the photometric data to the model, convolved with a circular Gaussian seeing disk of appropriate FWHM, using a $\chi^{2}$ minimization. The fitted parameters are: the two surface brightnesses $I_{\mathrm{e}}$ and $I_{\mathrm{d}}(0)$ (or $\mu_{\mathrm{e}}$ and $\mu_{\mathrm{d}}$ when given in magnitudes), the two scale lengths $r_{\mathrm{e}}$ and $r_{\mathrm{d}}$, the bulge ellipticity $\epsilon_{\mathrm{b}}$, and the system inclination $i$. Because of the difficulty inherent in estimating a luminosity distribution of unknown form (bulge) which is partially embedded in another (disk), we did not attempt to fit the bulge index $n$ explicitly (cf. Andredakis et al. 1995). Instead, for each galaxy the bulge was modeled with four different values of the exponent $n: 1,2,3$, and 4 ( $n=4$ for a de Vaucouleurs bulge). We then assessed the "quality" of the results for each set of 2-D fits, and determined the value of $n$ that gave the best all-round fit, thus assigning, in effect, a shape index to the bulge. Three quality indicators were considered in the assessment: the successful convergence of the process, the value of $\chi^{2}$, and the mean absolute residual.
When both $J$ and $K$ data were available, tests showed that bulge and disk decompositions and colors of the components were more stable when the two bands were fitted simultaneously. We also found no evidence for systematic scale length changes with wavelength of either component (cf., Evans 1994). Hence, when two bands were available, we performed a single fit keeping scale lengths, inclination, and bulge ellipticity the same for both bands, and letting the surface brightnesses of both components vary independently, but with constant color indexes $(J-K)_{\mathrm{b}}$ and $(J-K)_{\mathrm{d}}$.

The decomposition technique was extensively tested on a set of synthetic maps of axisymmetric bulge+disk distributions, covering a range of values for $i, \epsilon_{\mathrm{b}}$, and $B / D$ ratio similar to ours. We adopted an exponential thin disk, and generalized exponential laws for the bulge. An appropriate amount of noise was added to simulate the typical signal-to-noise levels we achieve, and finally the models were convolved with a range of PSF's to simulate the effects of seeing. The 2-D decomposition method was always able to recover the true parameter set, within the estimated errors, independently of the starting point in parameter space.

We also performed a 1-D fit to the radial profile, obtained by averaging along elliptical annuli; center and position angle of the annuli were held fixed, while the ellipticity was allowed to vary with semi-major axis. In the 1-D case, the convolution of the model with the PSF requires $i$ and $\epsilon_{\mathrm{b}}$ to be assigned a priori: as customary in $1-\mathrm{D}$ techniques, $\epsilon_{\mathrm{b}}$ was fixed at 0 and $i$ was determined from the outer $J$-band isophotes. The best-fit bulge index $n$ was determined in the same way as for the $2-\mathrm{D}$ models. 1- and 2-D techniques are compared in Sect. 4.1. 
The lower left panels in Fig. 1 show the results of the 2-D decompositions in terms of cuts along the semi-major axis ( $K$ band). Table 2 gives the best-fit parameters, including the shape index $n$ : the first line reports the values from the 2-D fit, the second reports the 1-D ones, and the third gives the values from the non-parametric decompositions (Sect. 3.2). Bulge and disk surface brightnesses are corrected to face-on values assuming optical transparency with $C=1$ :

$\mu_{\mathrm{d}}^{\mathrm{c}}=\mu_{\mathrm{d}}-2.5 C \log (\cos i)$,

$\mu_{\mathrm{e}}^{\mathrm{c}}=\mu_{\mathrm{e}}-2.5 C \log \left(1-\epsilon_{\mathrm{b}}\right)$,

and are denoted by $\mu_{\mathrm{e}}^{\mathrm{c}}(K)$ and $\mu_{\mathrm{d}}^{\mathrm{c}}(K)$ respectively. Table 3 lists the derived luminosities and bulge-to-disk ratios $(B / D)$; the first line reports the 2 -D parametric fit values, and the second gives the non-parametric values (see Sect. 3.2). The 1-D fixed-position-angle profiles together with Tables 2 and 3 are available in electronic form on the ftp node: sisifo.arcetri.astro.it in directory ftp/pub/nir.

\subsection{The non-parametric decomposition}

Kent (1986) was the first to introduce a non-parametric $(n p)$ method to separate bulge and disk; recently this has been extended to the profile analysis of 2-D images by Andredakis et al. (1995). We propose here a new, completely 2-D technique. Assuming the apparent ellipticities of the two components, $\epsilon_{\mathrm{b}}$ and $\epsilon_{\mathrm{d}}=1-\cos i$, are known, let us consider the average surface brightness of an annulus of ellipticity, say, $\epsilon_{\mathrm{d}}$. For a sufficiently narrow annulus, $A$, the disk contribution will be $I_{\mathrm{d}}(a)$, where $a$ is the semimajor axis. The total average brightness will be:

$<I(a)>_{\mathrm{d}}=I_{\mathrm{d}}(a)+\frac{1}{S_{\mathrm{A}}} \int_{A} I_{\mathrm{b}} \mathrm{d} S$

where $S_{\mathrm{A}}$ is the annular area. An analogous equation can be written for an annulus of ellipticity $\epsilon_{\mathrm{b}}$. The integral in Eq. (5) can be written as:

$\frac{2 S_{\mathrm{A}}}{\pi} \int_{0}^{\pi / 2} I_{\mathrm{b}}\left(a \sqrt{\cos ^{2} \theta+\frac{\rho_{\mathrm{d}}^{2}}{\rho_{\mathrm{b}}^{2}} \sin ^{2} \theta}\right) \mathrm{d} \theta$

where $\rho_{\mathrm{b}}$ and $\rho_{\mathrm{d}}$ are the axial ratios of the components. We then obtain for any $a$, a system of integral equations:

$$
\left\{\begin{aligned}
I_{\mathrm{b}}(a) & =<I(a)>_{\mathrm{b}} \\
& -\frac{2}{\pi} \int_{0}^{\pi / 2} I_{\mathrm{d}}\left(a \sqrt{\cos ^{2} \theta+\frac{\rho_{\mathrm{b}}^{2}}{\rho_{\mathrm{d}}^{2}} \sin ^{2} \theta}\right) \mathrm{d} \theta \\
I_{\mathrm{d}}(a) & =<I(a)>_{\mathrm{d}} \\
& -\frac{2}{\pi} \int_{0}^{\pi / 2} I_{\mathrm{b}}\left(a \sqrt{\cos ^{2} \theta+\frac{\rho_{\mathrm{d}}^{2}}{\rho_{\mathrm{b}}^{2}} \sin ^{2} \theta}\right) \mathrm{d} \theta
\end{aligned}\right.
$$

To solve the system we first compute $<I(a)>_{\mathrm{b}}$ and $<I(a)>_{\mathrm{d}}$ for two sets of annuli of ellipticities $\epsilon_{\mathrm{b}}$ and $\epsilon_{\mathrm{d}}$, respectively, each set sampling the entire light distribution. The two equations can then be solved iteratively for $I_{\mathrm{b}}$ and $I_{\mathrm{d}}$ at each $a$, starting with $\langle I\rangle_{\mathrm{b}}$ and $\langle I\rangle_{\mathrm{d}}$ as initial guesses to evaluate the integrals.

When defining the two sets of annuli, a proper sampling of the semi-major axis is of crucial importance. A reliable estimate of the integrals in Eq. (7) requires that each annulus of either set intersects with a sufficient number of annuli of the other set. If we constrain this number to be the same for every ellipse, the sampling is linear in $\log a$, i.e. the annuli become wider with increasing radius.

Results of the $n p$ decomposition technique are given in the third line of Table 2, and in the second line of Table 3. The non-parametric parameters are defined as follows. The bulge $r_{\mathrm{e}}$ is the semimajor axis of the ellipse enclosing half light; $\mu_{\mathrm{e}}$ is the bulge surface brightness at $r_{\mathrm{e}} ; r_{\mathrm{d}}$ is the semimajor axis of the ellipse enclosing 0.264 of the disk light, as in the exponential case. Since the central disk is not tightly constrained by the $n p$ fits, $\mu_{\mathrm{d}}$ is the central brightness of an exponential disk having scale length $r_{\mathrm{d}}$ and the same total luminosity as the $n p$ disk. The $n p$ colors of the components are derived by the total component luminosities in the two bands.

\subsubsection{Testing of non-parametric decomposition}

The $n p$ decomposition algorithm was tested on the same set of synthetic maps used for the parametric tests. Since the method applies only to components with different apparent ellipticity, we only tested models with $\epsilon_{\mathrm{b}}$ at least $10 \%$ smaller than $\epsilon_{\mathrm{d}}$. Under these conditions, to within the noise level, the method always proved to be able to recover the true bulge and disk distributions given the correct values of the ellipticities.

When errors on the ellipticities are introduced, the shape of the resulting components is affected in a systematic way: the value of $\epsilon_{\mathrm{b}}$ affects mainly the inner part of the disk, while $\epsilon_{\mathrm{d}}$ determines the shape of the outer bulge. In particular, if $\epsilon_{\mathrm{b}}$ is underestimated the inner disk is too steep, whereas if the bulge is too elliptical the inner disk develops a hole which gets deeper as $\epsilon_{\mathrm{b}}$ increases. If $\epsilon_{\mathrm{d}}$ is too small the outer bulge is too steep and viceversa. Although qualitatively these are expected trends, they are difficult to quantify in practice, since they depend on the shapes of the distributions, on the ellipticities, and on the seeing. Especially when the two components have similar ellipticities, even small errors on $\epsilon_{\mathrm{b}}$ and $\epsilon_{\mathrm{d}}(<0.1)$ perceptibly affect parameters such as the luminosity of the components or the $B / D$ ratio, as well as the shape of the profiles.

\subsubsection{Seeing corrections}

Simulations show that, if no correction is applied, seeing significantly affects $n p$ decompositions. The effects are 
Table 2. Decomposition results: structural parameters

\begin{tabular}{|c|c|c|c|c|c|c|c|c|c|c|}
\hline $\begin{array}{l}\text { Name } \\
(1)\end{array}$ & $\begin{array}{c}\mu_{\mathrm{e}}^{\mathrm{c}}(K) \\
\quad(2)\end{array}$ & $\begin{array}{c}(J-K)_{\mathrm{b}} \\
(3)\end{array}$ & $\begin{array}{c}r_{\mathrm{e}}(\mathrm{kpc}) \\
\quad(4)\end{array}$ & $\begin{array}{l}\epsilon_{\mathrm{b}} \\
(5)\end{array}$ & $\begin{array}{l}\mu_{\mathrm{d}}^{\mathrm{c}}(K) \\
\quad(6)\end{array}$ & $\begin{array}{c}(J-K)_{\mathrm{d}} \\
(7)\end{array}$ & $\begin{array}{c}r_{\mathrm{d}}(\mathrm{kpc}) \\
\quad(8)\end{array}$ & $\begin{array}{c}i \\
(9)\end{array}$ & $\begin{array}{c}\chi_{\nu}^{2} \\
(10)\end{array}$ & $\begin{array}{c}n \\
(11)\end{array}$ \\
\hline N 1024 & $\begin{array}{l}17.33(0.04) \\
13.50(0.03) \\
17.27\end{array}$ & & $\begin{array}{l}3.16(0.13) \\
0.92(0.03) \\
3.42\end{array}$ & $0.36(0.01)$ & $\begin{array}{l}18.85(0.14) \\
17.97(0.17) \\
18.38\end{array}$ & & $\begin{array}{l}8.21(0.49) \\
7.48(1.22) \\
5.72\end{array}$ & $\begin{array}{l}66.7(1.2) \\
68.9 \\
69.3\end{array}$ & $\begin{array}{l}1.2 \\
1.7 \\
1.0\end{array}$ & $\begin{array}{l}3 \\
1\end{array}$ \\
\hline N 2639 & $\begin{array}{l}18.84(0.07) \\
13.49(0.17) \\
15.78\end{array}$ & $\begin{array}{l}0.84 \\
0.86 \\
0.79\end{array}$ & $\begin{array}{l}6.42(0.18) \\
0.45(0.04) \\
1.16\end{array}$ & $0.32(0.01)$ & $\begin{array}{l}16.11(0.06) \\
15.62(0.07) \\
15.06\end{array}$ & $\begin{array}{l}0.98 \\
0.93 \\
0.91\end{array}$ & $\begin{array}{l}3.38(0.03) \\
3.25(0.08) \\
3.65\end{array}$ & $\begin{array}{l}65.2(0.3) \\
58.7 \\
60.8\end{array}$ & $\begin{array}{l}1.2 \\
4.2 \\
0.7\end{array}$ & $\begin{array}{l}4 \\
1\end{array}$ \\
\hline N 2775 & $\begin{array}{l}17.03(0.02) \\
18.11(0.10) \\
16.89\end{array}$ & $\begin{array}{l}0.96 \\
0.95 \\
1.07\end{array}$ & $\begin{array}{l}1.85(0.02) \\
4.07(0.43) \\
1.97\end{array}$ & $0.10(0.01)$ & $\begin{array}{l}16.77(0.02) \\
17.08(0.20) \\
15.95\end{array}$ & $\begin{array}{l}1.01 \\
1.13 \\
0.90\end{array}$ & $\begin{array}{l}4.60(0.03) \\
4.93(0.63) \\
4.51\end{array}$ & $\begin{array}{l}37.1(0.4) \\
29.5 \\
42.4\end{array}$ & $\begin{array}{l}1.4 \\
4.8 \\
1.0\end{array}$ & $\begin{array}{l}3 \\
4\end{array}$ \\
\hline N 2841 & $\begin{array}{l}15.65(0.02) \\
16.19(0.08) \\
15.62\end{array}$ & $\begin{array}{l}1.07 \\
1.06 \\
0.94\end{array}$ & $\begin{array}{l}0.86(0.01) \\
1.61(0.10) \\
0.86\end{array}$ & $\begin{array}{l}0.47(0.01) \\
0.30\end{array}$ & $\begin{array}{l}16.73(0.02) \\
17.40(0.12) \\
16.15\end{array}$ & $\begin{array}{l}1.04 \\
1.03 \\
1.10\end{array}$ & $\begin{array}{l}4.91(0.04) \\
6.67(0.55) \\
4.06\end{array}$ & $\begin{array}{l}59.8(0.2) \\
63.9 \\
64.7\end{array}$ & $\begin{array}{l}7.8 \\
6.3 \\
1.2\end{array}$ & $\begin{array}{l}2 \\
3\end{array}$ \\
\hline N 3593 & $\begin{array}{l}15.12(0.03) \\
13.67(0.07) \\
17.28\end{array}$ & $\begin{array}{l}1.38 \\
1.35 \\
0.86\end{array}$ & $\begin{array}{l}0.40(0.01) \\
0.43(0.03) \\
0.61\end{array}$ & $\begin{array}{l}0.26 \\
0.30\end{array}$ & $\begin{array}{l}16.98(0.03) \\
18.20(0.48) \\
15.68\end{array}$ & $\begin{array}{l}0.87 \\
0.71 \\
0.95\end{array}$ & $\begin{array}{l}2.11(0.02) \\
2.40(0.80) \\
1.51\end{array}$ & $\begin{array}{l}69.2(0.2) \\
70.1 \\
69.5\end{array}$ & $\begin{array}{l}10.4 \\
30.2 \\
0.8\end{array}$ & $\begin{array}{l}1 \\
1\end{array}$ \\
\hline N 3898 & $\begin{array}{l}15.33(0.02) \\
16.50(0.15) \\
15.84\end{array}$ & $\begin{array}{l}1.10 \\
1.09 \\
1.03\end{array}$ & $\begin{array}{l}0.90(0.01) \\
2.21(0.28) \\
0.90\end{array}$ & $\begin{array}{l}0.22 \\
0.25\end{array}$ & $\begin{array}{l}17.34(0.03) \\
18.27(0.70) \\
15.89\end{array}$ & $\begin{array}{l}0.81 \\
0.65 \\
0.82\end{array}$ & $\begin{array}{l}4.10(0.06) \\
4.37(1.75) \\
3.17\end{array}$ & $\begin{array}{l}59.7(0.4) \\
58.0 \\
58.5\end{array}$ & $\begin{array}{l}5.1 \\
7.3 \\
0.9\end{array}$ & $\begin{array}{l}2 \\
3\end{array}$ \\
\hline N 4378 & $\begin{array}{l}17.09(0.03) \\
17.62(0.15) \\
16.19\end{array}$ & $\begin{array}{l}0.86 \\
0.79 \\
0.92\end{array}$ & $\begin{array}{l}2.22(0.04) \\
2.94(0.42) \\
1.33\end{array}$ & $\begin{array}{l}0.18(0.01) \\
0.15\end{array}$ & $\begin{array}{l}17.62(0.04) \\
17.06(0.24) \\
15.56\end{array}$ & $\begin{array}{l}0.88 \\
1.16 \\
0.78\end{array}$ & $\begin{array}{l}4.94(0.08) \\
3.68(0.38) \\
3.67\end{array}$ & $\begin{array}{l}35.5(0.4) \\
36.9 \\
37.9\end{array}$ & $\begin{array}{l}1.2 \\
3.3 \\
1.8\end{array}$ & $\begin{array}{l}3 \\
4\end{array}$ \\
\hline N 4419 & $\begin{array}{l}16.58(0.03) \\
17.14(0.31) \\
15.02\end{array}$ & $\begin{array}{l}1.36 \\
1.25 \\
1.19\end{array}$ & $\begin{array}{l}1.01(0.03) \\
2.52(0.95) \\
0.39\end{array}$ & $\begin{array}{l}0.47(0.01) \\
0.25\end{array}$ & $\begin{array}{l}16.85(0.03) \\
17.20(0.59) \\
15.95\end{array}$ & $\begin{array}{l}0.90 \\
0.90 \\
1.01\end{array}$ & $\begin{array}{l}2.40(0.02) \\
2.44(0.33) \\
1.98\end{array}$ & $\begin{array}{l}73.7(0.2) \\
70.1 \\
72.1\end{array}$ & $\begin{array}{l}2.2 \\
25.0 \\
1.2\end{array}$ & $\begin{array}{l}3 \\
3\end{array}$ \\
\hline N 4450 & $\begin{array}{l}17.19(0.02) \\
17.71(0.13) \\
16.05\end{array}$ & $\begin{array}{l}0.95 \\
0.82 \\
1.24\end{array}$ & $\begin{array}{l}1.44(0.02) \\
1.89(0.23) \\
0.73\end{array}$ & $\begin{array}{l}0.25(0.01) \\
0.05\end{array}$ & $\begin{array}{l}17.32(0.02) \\
17.05(0.10) \\
15.85\end{array}$ & $\begin{array}{l}0.91 \\
1.04 \\
0.84\end{array}$ & $\begin{array}{l}4.67(0.04) \\
4.14(0.29) \\
3.18\end{array}$ & $\begin{array}{l}54.6(0.3) \\
55.2 \\
57.6\end{array}$ & $\begin{array}{l}2.7 \\
7.4 \\
2.7\end{array}$ & $\begin{array}{l}3 \\
4\end{array}$ \\
\hline N 4698 & $\begin{array}{l}15.91(0.03) \\
16.85(0.17) \\
16.29\end{array}$ & $\begin{array}{l}0.76 \\
0.89 \\
0.79\end{array}$ & $\begin{array}{l}0.58(0.01) \\
1.12(0.17) \\
0.96\end{array}$ & $0.24(0.01)$ & $\begin{array}{l}16.69(0.02) \\
17.69(0.20) \\
16.65\end{array}$ & $\begin{array}{l}0.96 \\
0.98 \\
0.99\end{array}$ & $\begin{array}{l}3.01(0.04) \\
4.59(0.72) \\
3.72\end{array}$ & $\begin{array}{l}41.5(0.4) \\
56.6 \\
54.5\end{array}$ & $\begin{array}{l}7.2 \\
15.7 \\
1.8\end{array}$ & $\begin{array}{l}2 \\
3\end{array}$ \\
\hline N 4845 & $\begin{array}{l}16.50(0.07) \\
15.22(0.14) \\
15.86\end{array}$ & $\begin{array}{l}1.54 \\
1.61 \\
1.43\end{array}$ & $\begin{array}{l}0.86(0.05) \\
0.59(0.05) \\
0.70\end{array}$ & $\begin{array}{l}0.23(0.02) \\
0.40\end{array}$ & $\begin{array}{l}18.05(0.04) \\
18.08(0.09) \\
17.92\end{array}$ & $\begin{array}{l}0.89 \\
0.91 \\
1.12\end{array}$ & $\begin{array}{l}5.26(0.10) \\
5.57(0.53) \\
4.59\end{array}$ & $\begin{array}{l}75.7(0.3) \\
75.5 \\
75.9\end{array}$ & $\begin{array}{l}6.1 \\
13.6 \\
1.3\end{array}$ & $\begin{array}{l}4 \\
2\end{array}$ \\
\hline N 5879 & $\begin{array}{l}18.92(0.05) \\
15.73(0.75) \\
16.00\end{array}$ & $\begin{array}{l}0.81 \\
0.94 \\
1.13\end{array}$ & $\begin{array}{l}2.45(0.05) \\
0.18(0.09) \\
0.31\end{array}$ & 0.25 & $\begin{array}{l}16.81(0.04) \\
15.92(0.07) \\
15.70\end{array}$ & $\begin{array}{l}0.87 \\
0.85 \\
0.74\end{array}$ & $\begin{array}{l}1.40(0.02) \\
1.24(0.05) \\
1.51\end{array}$ & $\begin{array}{l}75.8(0.3) \\
66.4 \\
65.7\end{array}$ & $\begin{array}{l}3.3 \\
9.6 \\
0.9\end{array}$ & $\begin{array}{l}3 \\
2\end{array}$ \\
\hline N 6314 & $\begin{array}{l}15.79(0.03) \\
18.55(0.40) \\
15.58\end{array}$ & $\begin{array}{l}1.16 \\
1.29 \\
1.04\end{array}$ & $\begin{array}{l}1.80(0.01) \\
12.2(5.06) \\
1.64\end{array}$ & 0.04 & $18.00(0.06)$ & 0.83 & $\begin{array}{l}8.50(0.01) \\
10.6(9.42) \\
6.05\end{array}$ & $\begin{array}{l}62.3(0.0) \\
70.0 \\
60.9\end{array}$ & $\begin{array}{l}2.5 \\
12.7 \\
1.1\end{array}$ & $\begin{array}{l}2 \\
3\end{array}$ \\
\hline I 724 & $\begin{array}{l}16.86(0.03) \\
16.60(0.30) \\
16.59\end{array}$ & $\begin{array}{l}1.40 \\
1.58 \\
1.12\end{array}$ & $\begin{array}{l}3.19(0.10) \\
2.25(0.71) \\
2.65\end{array}$ & 0.08 & $\begin{array}{l}18.46(0.06) \\
17.19(0.31) \\
17.84\end{array}$ & $\begin{array}{l}0.94 \\
0.92 \\
1.14\end{array}$ & $\begin{array}{l}11.4(0.44) \\
6.05(0.99) \\
10.7\end{array}$ & $\begin{array}{l}73.1(0.7) \\
67.7 \\
70.5\end{array}$ & $\begin{array}{l}2.0 \\
7.2 \\
1.4\end{array}$ & $\begin{array}{l}2 \\
2\end{array}$ \\
\hline
\end{tabular}

Line 1: 2-D parametric decompositions.

Line 2: 1-D parametric decompositions.

Line 3: $K$-band 2-D non-parametric decompositions.

stronger in the inner parts, the main feature being a central hole in the disk, whose extension is proportional to the seeing width. In fact, the seeing makes inner isophotes rounder, causing the component of higher ellipticity to be depressed. The disk profile, however, can be corrected by extrapolating the outer points to the center.

To recover the intrinsic bulge profile, we introduced a second iterative algorithm which proved to be quite effective on synthetic maps. The effect of seeing is accounted for by defining a correction coefficient $k_{\mathrm{b}}(a)$, for each point of the bulge profile, as the ratio of the convolved bulge distribution and an estimate of the true one. Each decomposition is performed using $k_{\mathrm{b}}(a)$ to degrade the assumed true distribution. After decomposition, the coefficients are recomputed by convolving the extracted bulge profile with the PSF, and a new decomposition is performed until both the coefficients and the profiles converge. The initial estimate of $k_{\mathrm{b}}(a)$ is provided by the convolution of the bulge profile extracted without any correction. 


\subsubsection{Estimate of the ellipticities}

To determine the apparent ellipticity of the components, we extracted from each image a radial profile of the ellipticity by fitting isophotes of variable ellipticity and fixed position angle. We then selected the lowest value beyond one seeing disk from the center as $\epsilon_{\mathrm{b}}$, and the average value in the outer parts as $\epsilon_{\mathrm{d}}$ (see also Andredakis et al. 1995). The values for the $J$ and $K$ bands were then averaged to obtain a single value of $\epsilon_{\mathrm{b}}$ and $\epsilon_{\mathrm{d}}$ for each galaxy. The accuracy of these estimates is difficult to assess: bright bulges can affect the isophotes even at large radii, leading to an underestimate of $\epsilon_{\mathrm{d}}$, while bars and spiral arms may often lead to overestimates. In turn, $\epsilon_{\mathrm{b}}$ can be easily underestimated because of the seeing. From simulations, it seems that a reasonable estimate for the uncertainty in $\epsilon_{\mathrm{d}}$ is about 0.1 ; it is certainly worse in $\epsilon_{\mathrm{b}}, 0.2$ or more in the worst cases.

We note that comparing the observed image to the extracted bulge+disk distribution, for example in terms of residuals, is of little use to test the ellipticities, since it is often possible to find excellent agreement for a wide range of $\epsilon_{\mathrm{b}}$ and $\epsilon_{\mathrm{d}}$. A more stringent constraint is provided by the color profiles of bulge and disk, which turn out to be quite sensitive to the values of $\epsilon_{\mathrm{b}}$ and $\epsilon_{\mathrm{d}}$. This has ultimately allowed us to verify the ellipticities a posteriori (in particular $\epsilon_{\mathrm{b}}$ ) by letting them vary within the estimated errors and checking the plausibility of the resulting color profiles. The final accuracy is estimated to be better than 0.05 .

\section{Results}

\subsection{Accuracy and comparison of the different decomposition methods}

As Fig. 1 clearly shows, the elliptically-averaged profiles reproduce quite well the major-axis cut in almost all cases. In fact, our sample contains only moderately inclined systems $\left(i<75^{\circ}\right)$, whose isophotes are reasonably elliptical. However, a comparison between the 1-D and 2-D results from Table 2 shows that the 1-D decomposition is always less accurate, and sometimes inconsistent with the 2-D one. The inferior quality of the 1-D fits is attested to both by the larger errors on the parameters and by the larger values of $\chi^{2}$. A comparison between $1-\mathrm{D}$ and $2-\mathrm{D}$ results for the galaxies with significant non-axisymmetric structure suggests that 2-D fits manage to reproduce the whole distribution, whereas 1-D decompositions tend to match closely only the inner part of the profile, i.e. the less noisy one (see also Byun \& Freeman 1995). Because of their superiority, when discussing parametric models, we will consider only the results of $2-\mathrm{D}$ decompositions.

No systematic differences are found between the various fitted parameters: the 1-D bulge and disk scale lengths
Table 3. Decomposition results: $K$-band integrated luminosities and bulge-to- disk ratios

\begin{tabular}{|c|c|c|c|c|c|}
\hline $\begin{array}{c}\text { Name } \\
(1)\end{array}$ & $\begin{array}{l}M_{\mathrm{b}} \\
(2)\end{array}$ & $\begin{array}{l}M_{\mathrm{d}} \\
(3)\end{array}$ & $\begin{array}{l}M_{\mathrm{g}} \\
(4)\end{array}$ & $\begin{array}{c}(B / D)_{K} \\
(5)\end{array}$ & $\begin{array}{c}(B / D)_{J} \\
\quad(6)\end{array}$ \\
\hline \multirow[t]{2}{*}{ NGC 1024} & -24.98 & -24.29 & -25.44 & & 1.89 \\
\hline & -25.17 & -23.83 & -25.45 & & 3.43 \\
\hline \multirow[t]{2}{*}{ NGC 2639} & -25.16 & -25.09 & -25.88 & 1.21 & 1.06 \\
\hline & -23.84 & -25.56 & -25.76 & 0.23 & 0.20 \\
\hline \multirow[t]{2}{*}{ NGC 2775} & -24.12 & -25.12 & -25.48 & 0.41 & 0.40 \\
\hline & -24.37 & -24.78 & -25.35 & 0.59 & 0.68 \\
\hline \multirow[t]{2}{*}{ NGC 2841} & -23.63 & -25.30 & -25.51 & 0.21 & 0.21 \\
\hline & -23.73 & -24.94 & -25.25 & 0.38 & 0.33 \\
\hline \multirow[t]{2}{*}{ NGC 3593} & -22.16 & -23.21 & -23.56 & 0.24 & 0.38 \\
\hline & -21.53 & -23.47 & -23.64 & 0.18 & 0.17 \\
\hline \multirow[t]{2}{*}{ NGC 3898} & -24.04 & -24.30 & -24.93 & 0.60 & 0.79 \\
\hline & -23.77 & -24.43 & -24.90 & 0.45 & 0.54 \\
\hline \multirow[t]{2}{*}{ NGC 4378} & -24.46 & -24.41 & -25.19 & 1.06 & 1.04 \\
\hline & -23.91 & -24.65 & -25.10 & 0.45 & 0.50 \\
\hline \multirow[t]{2}{*}{ NGC 4419} & -23.27 & -23.62 & -24.21 & 0.47 & 0.72 \\
\hline & -22.49 & -23.92 & -24.18 & 0.23 & 0.27 \\
\hline \multirow[t]{2}{*}{ NGC 4450} & -23.40 & -24.59 & -24.91 & 0.32 & 0.34 \\
\hline & -22.90 & -24.45 & -24.69 & 0.17 & 0.24 \\
\hline \multirow[t]{2}{*}{ NGC 4698} & -22.50 & -24.27 & -24.46 & 0.24 & 0.20 \\
\hline & -23.22 & -23.94 & -24.39 & 0.62 & 0.51 \\
\hline \multirow[t]{2}{*}{ NGC 4845} & -23.15 & -24.12 & -24.49 & 0.23 & 0.41 \\
\hline & -23.08 & -24.03 & -24.41 & 0.31 & 0.42 \\
\hline \multirow[t]{2}{*}{ NGC 5879} & -22.83 & -22.49 & -23.42 & 1.44 & 1.37 \\
\hline & -20.72 & -23.25 & -23.35 & 0.07 & 0.10 \\
\hline \multirow[t]{2}{*}{ NGC 6314} & -25.09 & -25.21 & -25.91 & 0.65 & 0.90 \\
\hline & -24.89 & -25.39 & -25.92 & 0.55 & 0.64 \\
\hline \multirow[t]{2}{*}{ IC 724} & -25.26 & -25.40 & -26.09 & 0.59 & 0.88 \\
\hline & -25.05 & -25.58 & -26.10 & 0.63 & 0.61 \\
\hline
\end{tabular}

Line 1: 2-D parametric decompositions.

Line 2: 2-D non-parametric decompositions.

are, to within the scatter, the same as those of the 2-D fits, and the fitted inclination of the 2-D model corresponds, on average, to the fixed value determined from the outer isophotes (and used in the 1-D fits). However, disks are definitely better constrained than bulges: the rms difference of the 1 - and 2-D $\mu_{\mathrm{d}}$ 's $(0.6 K$-mag) is half that for $\mu_{\mathrm{e}}(1.1 K-\mathrm{mag})$, and the dispersion in the ratio of $1-$ and $2-\mathrm{D} r_{\mathrm{e}}$ 's is $80 \%$, while that for the $r_{\mathrm{d}}$ 's is four times lower (21\%), comparable to the discrepancies among disk scale lengths from different authors found by Knapen \& van der Kruit (1991).

We have compared the results of our non-parametric $(n p)$ decompositions with the 2-D parametric ones, and with those of Kent (1988). There is no systematic difference between our 2-D and $n p$ bulge and disk luminosities, as the mean difference is $-0.4 \pm 0.6 \mathrm{mag}$ for $M_{\mathrm{b}}$, and $-0.1 \pm 0.4$ for the disk. Again, disk parameters are more consistent than those of the bulge. Parametric ellipticities are not significantly different from $n p$ ones: the mean ratio of the $n p \epsilon_{\mathrm{b}}$ 's and those fitted by the $2-\mathrm{D}$ parametric 


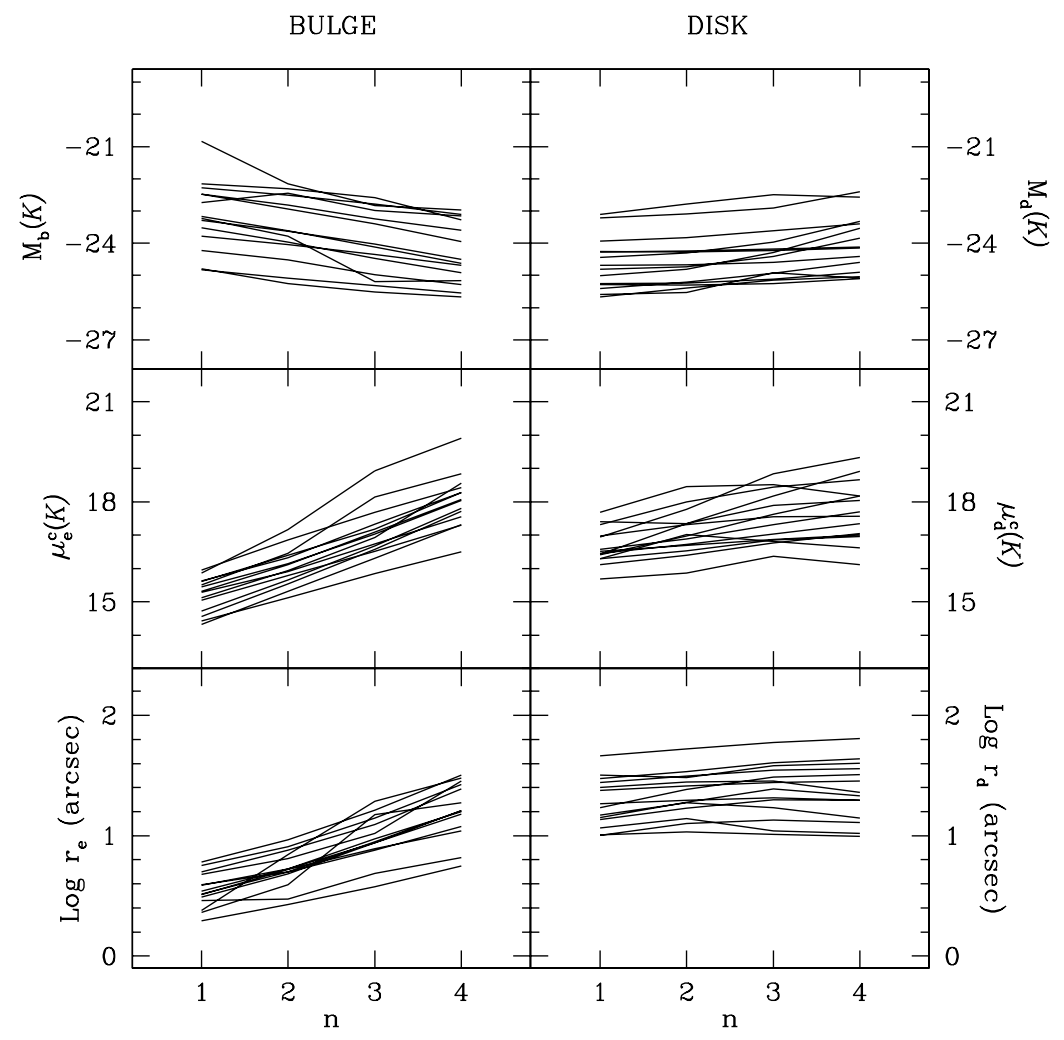

Fig. 2. Bulge and disk fitted parameter variation with $n$. Bulge parameters are shown in the left panels: from top to bottom, absolute $K$-magnitude $M_{\mathrm{b}}(K)$, effective surface brightness $\mu_{\mathrm{e}}^{\mathrm{c}}(K)$, and effective scale length $r_{\mathrm{e}}$. Analogous disk parameters are shown in the right panels. The curves show, for a given galaxy, fit parameters obtained with $n$ going from 1 to $4 . \mu_{\mathrm{e}}^{\mathrm{c}}(K)$ and $\mu_{\mathrm{d}}^{\mathrm{c}}(K)$ are corrected to face-on and in units of mag $\operatorname{arcsec}^{-2}$. Scale lengths are in $\operatorname{arcsec}$

method is $1.1 \pm 0.5$. System inclinations agree very well, with a mean ratio of $0.97 \pm 0.09$. Also, the bulge ellipticities determined by Kent (1988) are about the same as those used in our $n p$ decomposition, but with large scatter; the inclinations are in good agreement, with a mean ratio of $1.01 \pm 0.09$.

\subsection{Dependence on $n$ of parametric components}

In order to compare our results with those of other authors, we have investigated trends with $n$ of the bulge and disk fitted parameters. Figure 2 shows the systematic variation in these, for a given galaxy, as a function of $n$. It can be seen from the figure that the derived bulge parameters strongly depend on the form of the fitting function. The same bulge, when fitted with small $n$, appears to be "denser" (that is to say brighter $\mu_{\mathrm{e}}$ ), more compact (smaller $r_{\mathrm{e}}$ ), and less luminous than when fitted with large $n$. Quantitatively, the changes are dramatic as $n$ goes from 1 to 4 with a mean change in $\mu_{\mathrm{e}}$ of 3 mag $\operatorname{arcsec}^{-2}$, and in scale length of roughly a factor of 3 . Moreover, the dispersion in the fitted values also increases with $n$; the spread of $\log r_{\mathrm{e}}$ at $n=4$ is 1.5 times larger than that at $n=1$, and the spread of $\mu_{\mathrm{e}}$ is more than twice as large.
The derived disk parameters also change with the $n$ of the bulge. $\mu_{\mathrm{d}}$ tends to be fainter for bulge $n$ larger, and, as for the bulge, the dispersion in $\mu_{\mathrm{d}}$ increases with $n$. The only parameter that is stable with $n$ is the disk scale length $r_{\mathrm{d}}$, although its dispersion does increase slightly with $n$.

We conclude that, at least statistically, bulge structural parameters are strongly influenced by the form of the function used to derive them. Not only are the parameters themselves altered by constraining the form of the bulge, but also the dispersion in the parameters is changed. Independently of the best-fit $n$, requiring a de Vaucouleurs law to fit the bulge yields more tenuous, extended, and luminous spheroids, together with wider distributions of the parameters (larger dispersion), than does using a simple exponential.

\subsection{Bulges}

In terms of the "quality indicators" mentioned in Sect. $3.1, n=3$ gave superior results for the majority of galaxies, while $n=2$ was the second-best choice. In only two cases $\operatorname{did} n=4$ give the highest quality fit, and in one case (NGC 3593) $n=1$. These values are in general agreement with the trend noted by Andredakis et al. (1995) who fit- 
Table 4. Statistics of parametric components

\begin{tabular}{llllc}
\hline & & Mean $(\sigma)$ & Median & Range \\
\hline \multirow{6}{*}{ Bulge } & $n$ & $2.6(0.8)$ & 3 & $1-4$ \\
& $r_{\mathrm{e}}$ & $1.9(1.6)$ & 1.6 & $0.4-6.4$ \\
& $\mu_{\mathrm{e}}^{\mathrm{c}}(K)$ & $16.7(1.1)$ & 16.8 & $15.1-18.9$ \\
& $\epsilon_{\mathrm{b}}$ & $0.24(0.13)$ & 0.23 & $0.04-0.50$ \\
& $(J-K)_{\mathrm{b}}$ & $1.13(0.27)$ & 1.06 & $0.72-1.55$ \\
& $M_{\mathrm{b}}(K)$ & $-23.9(1.0)$ & -23.8 & $-25.3--22.2$ \\
\hline \multirow{5}{*}{ Disk } & $r_{\mathrm{d}}$ & $4.9(2.8)$ & 4.6 & $1.40-11.4$ \\
& $\mu_{\mathrm{d}}^{\mathrm{c}}(K)$ & $17.3(0.78)$ & 17.1 & $16.1-18.9$ \\
& $(J-K)_{\mathrm{d}}$ & $0.96(0.08)$ & 0.94 & $0.85-1.11$ \\
& $M_{\mathrm{d}}(K)$ & $-24.4(0.8)$ & -24.3 & $-25.4--22.5$ \\
\hline
\end{tabular}

ted the $n$ of non-parametric bulge profiles and found that early-type spirals tend to have bulges with $n \sim 2-3$. We also note that, in our experience, it is usually difficult to determine the best $n$ with a precision much better than 1 .

The distribution of the best- $n$ bulge parameters is illustrated in the left panels of Fig. 3. The median bulge parameters are $\mu_{\mathrm{e}}^{\mathrm{c}}(K)=16.8 \mathrm{mag} \operatorname{arcsec}^{-2}$, and $r_{\mathrm{e}}=1.6$ $\mathrm{kpc}$, as reported in Table 4. Our bulges are more tenuous, larger, and more luminous than those of similar type in de Jong (1996a), as expected given the fixed $n=1$ used by him. The median apparent $\epsilon_{\mathrm{b}}$ is 0.24 from parametric decompositions and 0.25 in $n p$ decompositions. This translates into a median intrinsic ellipticity of 0.36 or 0.33 from $n p$ values. Notably, 0.33 is also the commonest intrinsic ellipticity found in elliptical galaxies, if they are assumed to be rotational ellipsoids, either oblate or prolate (Mihalas \& Binney 1981). In any case, bulges are rarely spherical and the results of studies assuming so should be treated with caution.

Correlations between (average) surface brightness and scale length have been found for spiral bulges (Kent 1985; Kodaira et al. 1986) and for ellipticals (Kormendy 1977; Hoessel \& Schneider 1985; Djorgovski \& Davis 1987), and these two observables constitute an almost face-on view of the "fundamental plane" (FP) (e.g., Kormendy \& Djorgovski 1989, and references therein). The left panels of Fig. 4 show scatter plots of bulge $\mu_{\mathrm{e}}^{\mathrm{c}}(K)$ and $\left\langle\mu^{\mathrm{c}}(K)\right\rangle_{\mathrm{e}}$ vs. $r_{\mathrm{e}},\left\langle\mu^{\mathrm{c}}(K)>_{\mathrm{e}}\right.$ is the average surface brightness within the half-light isophote commonly used in FP studies. The upper panel shows results for all values of $n$, and the lower one only best- $n$ values. Our results are consistent with the slope within the FP found for bulges by Andredakis et al. (1995), shown as a dashed line in the figure. Although we have transformed their regression line to our distance scale and to the $K$-band according to their precepts, we note a slight offset: our best- $n$ bulges are generally dimmer ( $\sim 0.5 \mathrm{mag}$ ), for a given $r_{\mathrm{e}}$, than theirs.

Figure 4 gives further insights as to what happens when the form of the bulge is constrained to one value of $n$. As discussed in the previous section, when for example $n$ is 4 , the resulting bulge parameters lie in the tenuous, extended portion of the parameter space; when $n$ is 1 , resulting bulges are smaller and denser (see also Fig. 2). From Fig. 4, it appears that different $n$ values, that is to say different bulge shapes, occupy different regions of the FP. Such a behavior is evident in both the upper- and lower-left graphs. An analysis of the correlation coefficients shows that while the best- $n$ set of points is significantly correlated, as is the global set of points for all values of $n$, each individual set with fixed $n$ is not. Moreover, the slopes of each fixed $n$ group increase with $n$, although even the slope of $n=4$ is not as large as the global one. The appearance of the top-left graph is determined mainly by a "geometrical" effect, that is a constant luminosity relation although, see Fig. 2, higher $n$ 's produce slightly more luminous bulges. Independently of the details, it is clear that the large scatter related to the uncertainty on the decomposition has a high incidence on the position of a bulge within the FP, as shown by the error bar in the lower-left panel.

It has been suggested that residuals relative to the FP are correlated with shape parameters (Hjorth \& Madsen 1995; Prugniel \& Simien 1997). Although this projection of the FP is not appropriate for such considerations, the lower-left graph (bulge best $n$ ) in Fig. 4 suggests that even the distribution within the FP is at least partially generated by form variations. The distribution of the bulge $n p$ parameters does not reveal any dramatically different behavior; if anything, the distribution is tighter and situated in the low- $n$ region of the plot.

\subsubsection{Bulge colors}

The median $(J-K)_{\mathrm{b}}$ of 1.06 (1.04 for the $n p$ decomposition) agrees with the colors measured by Giovanardi \& Hunt (1996), and is redder by about $0.1-0.2 \mathrm{mag}$ than those measured in later types (Frogel 1985; Giovanardi \& Hunt 1988). The scatter is large, $0.3 \mathrm{mag}$, with some bulges having $J-K$ as high as 1.5 (NGC 4845). We find that $(J-K)_{\mathrm{b}}$ correlates $\left(98 \%\right.$ significance) with $\mu_{\mathrm{e}}^{\mathrm{c}}(K)$, in the sense that redder colors are associated with "denser" bulges. In contrast, $(J-K)_{\mathrm{b}}$ is independent of $M_{\mathrm{b}}(K)$, and of total galaxy luminosity.

The four objects (NGC 3593, 4419, 4845, and IC 724) with $(J-K)_{\mathrm{b}}>1.3$ also show red extended circumnuclear structure in the color images of Fig. 1, inflections or bumps in their surface brightness profiles, and red gradients in the inner color profiles. Such features have been observed in starburst galaxies (Hunt et al. 1997), and we would argue that the red $J-K$ bulge color is revealing star formation in progress. The most clear-cut case is NGC 3593 which, besides a high mid-infrared $12 \mu \mathrm{m}$ surface brightness (Soifer et al. 1989) and high molecular gas content (Sage 1993), hosts two counterrotating stellar disks and a disk of ionized gas (Bertola et al. 1996). 
Bulge parameters

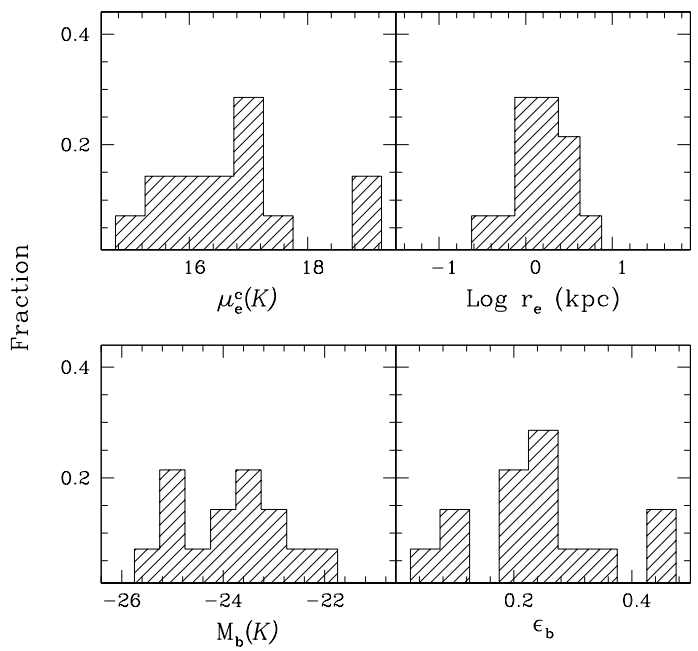

Disk parameters

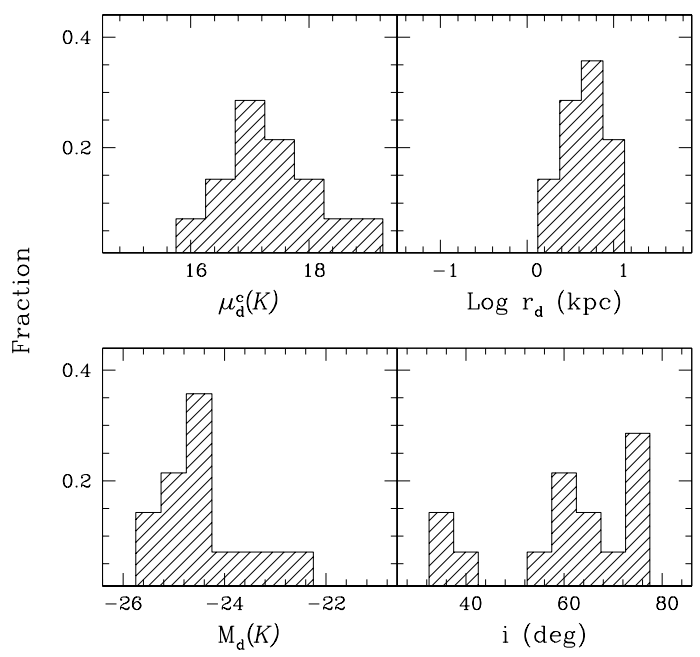

Fig. 3. Distributions of best- $n$ bulge and disk parameters. Surface brightnesses are corrected to face-on and in units of mag $\operatorname{arcsec}^{-2}$. Scale lengths are in kpc
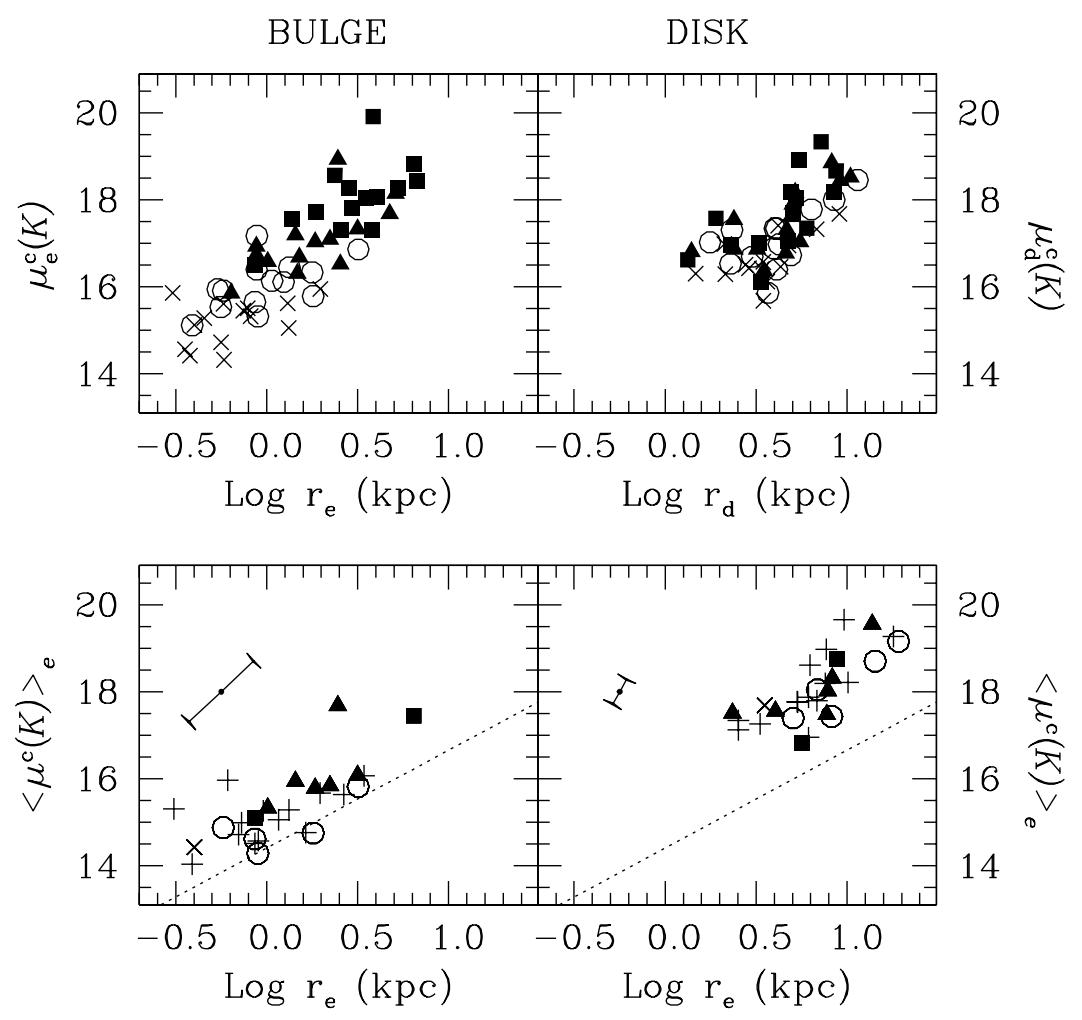

Fig. 4. Scatter plots of bulge and disk $\mu^{\mathrm{c}}(K)$ vs. $\log r$. Scale lengths are in kpc. The left panels show bulge parameters, and the right those of disks. The upper panels show points for all values of $n$, with $n=1,2,3,4$ shown by $\times$ 's, open circles, filled triangles, and filled squares, respectively. The lower panels show only the best- $n$ points, with $n$ denoted as in the upper panel, together with the non-parametric results shown as crosses. In the lower panels, $<\mu^{\mathrm{c}}(K)>_{\mathrm{e}}$ is the average surface brightness within the effective isophote; note that also for disks the abscissa is $r_{\mathrm{e}}$. In the lower panels, the dashed line shows the regression found by Andredakis et al. (1995) converted to our distance scale and to the $K$-band according to their precepts. The inclined error bars represent the average shift related to an uncertainty of \pm 1 in the bulge index $n$ 
Moreover, $\mathrm{H} \alpha$ images reveal an HII-region ring (Pogge \& Eskridge 1993) whose structure closely resembles that seen in our $J-K$ image. NGC 4419, the only barred galaxy in our sample, is a LINER (Huchra \& Burg 1992) with mid-infrared properties (Soifer et al. 1989; Devereux 1987) and CO content (Young et al. 1995) typical of starbursts. NGC 4845 was defined as a starburst by David et al. (1992) on the basis of its FIR-to-blue luminosity ratio and X-ray excess. IC 724, one of the most distant in our sample, harbors more than $10^{9} M_{\odot}$ of HI (Eder et al. 1991), but we have found no evidence in the literature for star formation activity. The $J-K$ image may be just revealing a normal bulge, partially obscured by a dusty disk.

\subsection{Disks}

The distribution of the disk parameters is shown in the right panels of Fig. 3. Similar to the results of de Jong (1996a) for early spiral types, the median disk has a $\mu_{\mathrm{d}}^{\mathrm{c}}(K)$ of $17.1 \mathrm{mag} \operatorname{arcsec}^{-2}, r_{\mathrm{d}}=4.6 \mathrm{kpc}$; with $M_{\mathrm{d}}(K)=$ $-24.3 \mathrm{mag}$ it is slightly more luminous than the median bulge. The median ratio of $r_{\mathrm{d}}$ and isophotal (optical) radius $R_{25}$ is 0.24 (shown in Fig. 5 as a dotted line in the upper left panel), comparable to what is found in late-type spirals (Giovanardi \& Hunt 1988; Giovanelli et al. 1995). Although similar in size, these early-type disks are more than $1 K$-mag $\operatorname{arcsec}^{-2}$ brighter than those in late-type spirals (Giovanardi \& Hunt 1988).

The right panels of Fig. 4 show scatter plots of disk $\mu_{\mathrm{d}}(K)$ vs. $r_{\mathrm{d}}$ (upper panel) and of disk $\left\langle\mu^{\mathrm{c}}(K)>_{\mathrm{e}}\right.$ vs. $r_{\mathrm{e}}$ (lower panel). As for the bulge, correlations of disk $\mu_{\mathrm{d}}^{\mathrm{c}}(K)$ with $r_{\mathrm{d}}$ have been noted for some time (e.g., Kent 1985). It is interesting to note that, when plotted in terms of the photometric observables commonly used in FP studies (lower-right panel), disks dwell in a region of this FP projection which is contiguous and similar in shape and extent to that of bulges. The disks appear to extend the bulge relation to larger radii and fainter surface brightnesses. Also evident, in the lower-right panel, is the rough consistency with the slope for bulges found by Andredakis et al. (1995), although with a large offset. It is clear from Fig. 4 that, unlike the bulge, the relation between disk parameters does not vary substantially with bulge $n$.

\subsubsection{Disk colors}

The median $(J-K)_{\mathrm{d}}$ of 0.94 (0.91 for the $n p$ decomposition) is similar to the central colors of late-type spirals (Frogel 1985; Giovanardi \& Hunt 1988). The scatter about the mean is $0.08 \mathrm{mag}$, smaller than for $(J-K)_{\mathrm{b}}$. The median disk is $0.12 \mathrm{mag}$ bluer than the bulge, an effect not noted by Terndrup et al. (1994) whose sample was dominated by later types. As for bulges, redder disks tend to be "denser" (98\% significance ), and $(J-K)_{\mathrm{d}}$ is independent of $M_{\mathrm{d}}$ and inclination.

\subsubsection{Opacity of the disks}

Although the diagnostics of dust content in galaxies have been extensively revised in recent years (e.g. Byun et al. 1994 - BFK; Bianchi et al. 1996), such studies have made clear that disk opacities are not easy to determine. In the following we gather the indications about disk opacity obtained here; none of the tests we adopt is particularly stringent, due mainly to the small number statistics, but all converge on conservative estimates for the opacity of early type disks: $\tau_{V}(0)$ ranges from 2 to 4 , where $\tau_{V}(0)$ is the central optical depth in the $V$ band (face-on). This is essentially the same result reached by Peletier \& Willner (1992) and Giovanardi \& Hunt (1996).

(a) Correlation between apparent disk brightness and inclination. We find a slight trend in both bands, with slopes $C_{J}=0.66 \pm 0.23$ and $C_{K}=0.73 \pm 0.23$, both compatible with a fully transparent disk (with $C=1$, see Eq. (4)). Taken at face value, a $C=0.7$ corresponds to a $\tau_{V}(0)$ of $\sim 1.1$ if measured in the $J$ band, and to $\tau_{V}(0) \simeq 1.8$ if in $K^{3}$. These moderate values for the central opacity imply that the spread observed in the NIR $\mu_{\mathrm{d}}^{\mathrm{c}}$ is intrinsic and not due to extinction; de Jong (1996b) reached a similar solution on the basis of a larger sample.

(b) Variation of disk scale length with wavelength and inclination (Evans 1994; Peletier et al. 1994). Five of the sample galaxies have been parametrically decomposed in the optical: either $r$ (Kent 1985; NGC 2639), $V$ (Kodaira et al. 1986; NGC 3898, 4698), or $B$ (Boroson 1981; NGC 2775, 2841, 3898). We find a trend in the ratios of our to their $r_{\mathrm{d}}$ : for the only measurement in $r$ the ratio is exactly 1 , it decreases to 0.85 in $V$, and to 0.70 in $B$. In addition, these ratios depend on the inclination, thus providing a test which is largely free from the influence of intrinsic color gradients. The correlation, in the sense of smaller ratios for higher $i$, implies $\tau_{V}(0) \leq 3$. These results are consistent with Peletier et al. (1994), who find that $B$ and $K$ scale length ratios vary from 1.2 to 2.0 , and with inclination.

(c) Colors. As noted in the previous section, $(J-K)_{\mathrm{d}}$ does not depend on $i$. We estimate the maximum $(3 \sigma)$ slope of $(J-K)_{\mathrm{d}}$ vs. sec $i$ which is still compatible with our data to be 0.075 . For a Triplex model (Disney et al. 1989 ) with $\zeta=0.5$ (Peletier \& Willner 1992), this implies a $\tau_{V}(0) \leq 3$. We noted in Sect. 4.4.1 that red disks were associated with bright $\mu_{\mathrm{d}}^{\mathrm{c}}$, which again points to moderate opacities. Indeed, since $\mu_{\mathrm{d}}^{\mathrm{c}}$ is corrected for inclination assuming transparency, a high opacity would translate into faint brightnesses for reddened disks.

${ }^{3}$ From Fig. 8 in BFK and assuming $A(\lambda) / A(V)$ as in Cardelli et al. (1989); these estimates have been corrected approximately for the lower dust albedo in the NIR. 


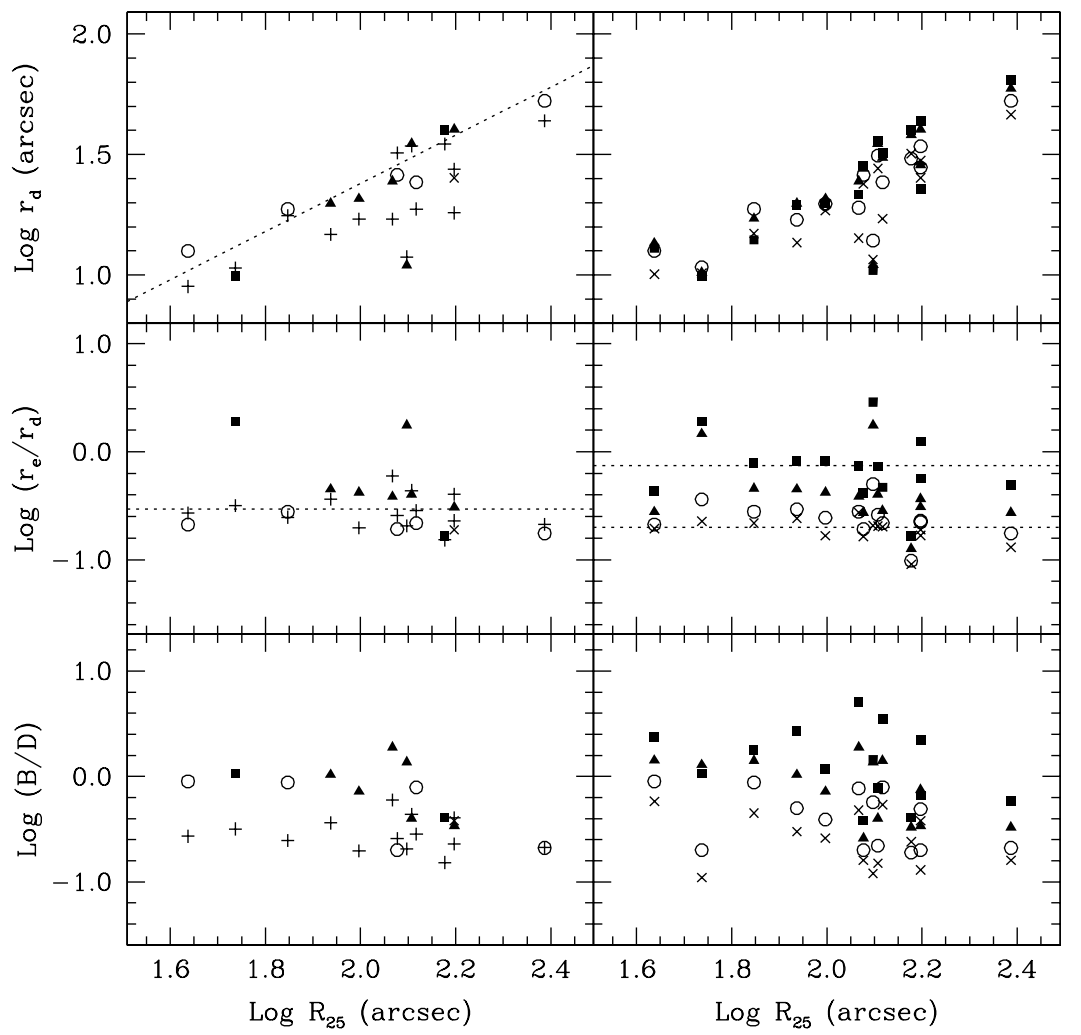

Fig. 5. Disk scale lengths, ratio of bulge and disk scale lengths, and $B / D$ vs. optical isophotal radius $R_{25}$. Best- $n$ values are shown in the left panels, and values for all $n$ are shown on the right; symbols are as in Fig. 4. The upper panels show $\log r_{\mathrm{d}}$ vs. $\log R_{25}$; the dotted line in the upper left panel illustrates a linear relationship between $r_{\mathrm{d}}$ and $R_{25}$ with a mean ratio $r_{\mathrm{d}} / R_{25}$ of 0.24 . The middle panels show the ratio $\log r_{\mathrm{e}} / r_{\mathrm{d}}$ vs. $\log R_{25}$; the dotted line on the left gives the best- $n$ median constant value $r_{\mathrm{e}} / r_{\mathrm{d}}=0.3$, while the pair of lines on the right show the $n=1$ median $r_{\mathrm{e}} / r_{\mathrm{d}}=0.2$, and the $n=4$ median $r_{\mathrm{e}} / r_{\mathrm{d}}=0.7$. The lower panels show $\log (B / D)$ vs. $\log R_{25}$

\subsection{Relationship between bulge and disk}

The best- $n$ median $B / D$ ratio is 0.8 with values ranging from 0.2 to 2 ; even in this early-type sample, more than two thirds of the galaxies have disks more luminous than bulges. With the exception of NGC $1024, B / D$ ratios obtained from the $n p$ decomposition are always less than 1 (as can be seen from the lower left panel in Fig. 5). The two methods yield $B / D$ values which differ by almost a factor of $\sim 2$, but with large scatter. We have verified that this is mainly imputable to the choice of $\epsilon_{\mathrm{b}}$ and $i$, the values adopted in the $n p$ case being lower. Our parametric $B / D$ ratios are comparable, although somewhat larger, to those (parametric) found by Kent (1985) in the $r$ band. Also, our $B / D$ 's (both parametric and $n p$ ) in the $K$ band are 10 $\sim 15 \%$ larger than in $J$. That the $K$-band $B / D$ is larger than in the optical was also noted by de Jong (1996a), but with values smaller than ours due to his choice of $n=1$ bulges. It is evident that the $B / D$ ratio is rather model dependent, and different decomposition methods provide estimates differing by factors of 2 or more, as illustrated in Fig. 5 where $\log r_{\mathrm{d}}, \log r_{\mathrm{e}} / r_{\mathrm{d}}$, and $\log B / D$ are shown as a function of optical (isophotal) radius ${ }^{4}$. Inspection of this figure also shows that the derived bulge and disk pa-

\footnotetext{
4 These were taken from Table 1.
}

rameters, including best $n$, are not appreciably affected by biases associated with galaxy apparent size.

A linear correlation between $r_{\mathrm{e}}$ and $r_{\mathrm{d}}$ over all spiral types has been recently found by de Jong (1996a) and Courteau et al. (1996). They interpret the correlation as an indication that the Hubble sequence is scale-free since the relative size of bulge and disk does not depend on morphological type. It can be seen from the middle panels that our data are also consistent with constant $r_{\mathrm{e}} / r_{\mathrm{d}}$; the sample median best- $n r_{\mathrm{e}} / r_{\mathrm{d}}$ of 0.3 is shown as a dotted line. This value is a factor of 2 larger than that found by Courteau et al. in the $r$ band, $\left\langle r_{\mathrm{e}} / r_{\mathrm{d}}\right\rangle=0.13$ and by de Jong for the $K^{\prime}$ data alone, $\left\langle r_{\mathrm{e}} / r_{\mathrm{d}}\right\rangle=0.14^{5}$. However, $r_{\mathrm{e}} / r_{\mathrm{d}}$ appears to be strongly influenced by the bulge parameterization: the dashed lines shown in the middle right panel of Fig. 5 illustrate the values obtained from our $n=1$ fits (sample median $r_{\mathrm{e}} / r_{\mathrm{d}}=0.2$ ), and for the $n=4$ fits (median $r_{\mathrm{e}} / r_{\mathrm{d}}=0.7$ ). According to whether bulges are fit with a simple exponential or with the de Vaucouleurs law, $r_{\mathrm{e}} / r_{\mathrm{d}}$ changes by more than a factor of 3 . Hence, if the best-fit $n$ changes with morphological type as suggested by Andredakis et al. (1995), the claims made by

\footnotetext{
${ }^{5}$ Their values have been converted to bulge effective scale lengths using the conversion factor for $n=1$ given in the Appendix.
} 
Courteau et al. for a scale-free Hubble sequence may be premature.

\subsection{Color gradients}

Since we adopt the same scale length in $J$ and $K$, our parametric decompositions yield bulges and disks with uniform color. On average, the resulting bulges are redder than the disks by more than $0.1 \mathrm{mag}$, and we should detect a significant color gradient at the transition between bulge and disk. Such gradients are clearly evident in NGC 3593, 3898, 4419, 4845, 6314 and IC 724, all objects whose bulge and disk colors differ greatly. When such gradients are present, they also appear, enhanced, in the $r-K$ profiles.

Regarding the gradients within the single components, we give no estimate of bulge color gradients ${ }^{6}$. For the disk, following Terndrup et al. (1994), we estimated outer (>3 kpc) color gradients, computed by fitting $J-K$ and $r-K$ versus $\log r$ (in kpc); they will be denoted with $\delta(J-K)$ and $\delta(r-K)$ respectively ${ }^{7}$. We detect $J-K$ gradients at $3 \sigma$ in only one case, NGC 6314 , and in six if we consider a $2 \sigma$ limit. All galaxies with $J-K$ gradients for which we have $r$ data, namely NGC 3593, 3898, 4378, 4419 and 6314 , also show significant $\delta(r-K)$. Significant $\delta(r-K)$ is also found in NGC 2639, 4845 and IC 724 . In agreement with de Jong (1996b), we only find negative gradients, ranging from -0.24 to $-0.47 \mathrm{mag}$ per decade in $J-K$, and from -0.26 to -1.03 in $r-K$. There is no correlation between $\delta(J-K)$ and inclination, but inclined galaxies have steeper $r-K$ profiles (see Fig. 6).

Such color gradients provide a last assessment of the disk opacity, already discussed in Sect. 4.4.2. While NIR colors are stable across the disk, we find a prevalence of negative trends in $r-K$, especially in inclined galaxies (see Fig. 6). A weighted fit of $\delta(r-K)$ vs. $-2.5 \log (\cos i)$ yields a slope of $-0.30 \pm 0.08$. In $B-I$, a common feature of the models (e.g. BFK) is that gradients tend to steepen with increasing $i$ only in rather transparent disks; for $\tau_{V}(0) \geq 3$ the trend is reversed due to a saturation effect. It has also been shown by Bianchi et al. (1996) that disk color gradients are not greatly influenced by the dust scattering properties. In $r-K$ the reddening will be larger by a factor of 2 for the same $\tau_{V}(0)$, so the observed slope is roughly indicative of a $\tau_{V}(0) \simeq 1.5(\mathrm{BFK}$; Bianchi 1995).

\footnotetext{
${ }^{6}$ This could be accomplished, in principle, using $n p$ profiles or the inner part of the obseved color profiles but they are difficult to detect. Due to the seeing, the very inner color profile is hardly reliable, while at outer radii the bulge brightness rapidly falls deep below the disk.

7 We used global profiles rather than $n p$ disk profiles because the latter are not available for $r-K$, and because the two types of profile are very similar in $J-K$.
}

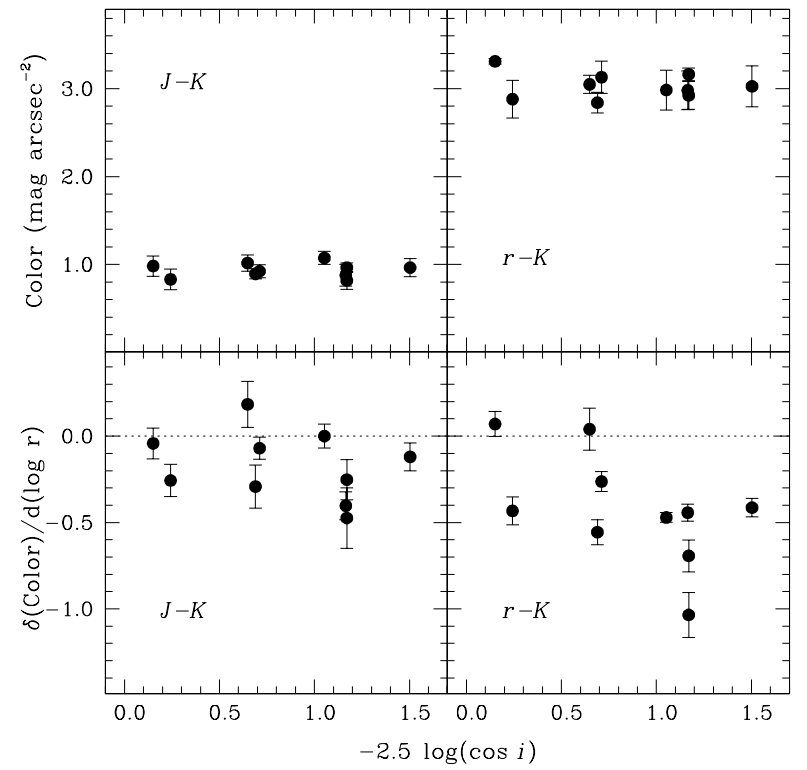

Fig. 6. Colors and color gradients vs. inclination. The upper panels show the mean of $J-K$ and $r-K$ colors beyond $3 \mathrm{kpc}$ from the center. Color gradients evaluated in the same region are shown in the lower panels

\section{Summary}

We have decomposed $J$ - and $K$-band images of 14 earlytype spirals into bulge and disk components. 2D nonparametric solutions and results from fitting a parametric model of generalized exponential $1 / n$ bulges and simple exponential disks are compared, and general characteristics of early-type spiral bulges and disks are examined. We find that:

1. Even using objective and refined techniques, the decomposition in structural components is far from being a robust and unique process. For the parametric methods, significantly different decompositions are obtained for different bulge distribution laws. Non-parametric techniques, on the other hand, appear to be affected by the choice of the ellipticities of the components which are difficult to evaluate objectively. Of the two components, the bulge is the most subject to errors, since the inner part is masked by seeing, and the outer regions are buried beneath the disk.

2. Bulge structural parameters are strongly influenced by the form of the function used to derive them. The same bulge, when fitted with small $n$, appears to be "denser" (brighter $\mu_{\mathrm{e}}$ ), more compact (smaller $r_{\mathrm{e}}$ ), and less luminous than when fitted with large $n$. The dispersion of the fitted parameters also increases with $n$.

3 . The median early-type bulge has a shape index $n$ between 2 and $3, \mu_{\mathrm{e}}^{\mathrm{c}}(K)=16.8 \mathrm{mag} \operatorname{arcsec}^{-2}$, and $r_{\mathrm{e}}=$ $1.6 \mathrm{kpc}$. It is also red, with $(J-K)_{\mathrm{b}}=1.06$, and redder bulges tend to be "denser", that is with brighter $\mu_{\mathrm{e}}$. 
4. As noted by Kent (1988), bulges are rarely spherical. The median intrinsic ellipticity is 0.34 , equivalent to a disk with $50^{\circ}$ declination. This restricts the applicability of non-parametric techniques to rather inclined systems and suggests treating with caution the studies which assume spherical bulges.

5. The median early-type disk with $\mu_{\mathrm{d}}^{\mathrm{c}}(K)=17.1$ is more than 1 mag $\operatorname{arcsec}^{-2}$ brighter than later-type disks, and bluer than the bulge in $(J-K)$ by more than $0.1 \mathrm{mag}$. Disk scale lengths agree fairly well with those found by other at different wavelengths, and we confirm a tendency for NIR disk scale lengths to be smaller than those at optical wavelengths (e.g., Peletier et al. 1994). $r_{\mathrm{d}} / R_{25}$ is approximately constant, 0.24 , similar to the value of 0.25 found for late-type spiral disks (Giovanardi \& Hunt 1988; Giovanelli et al. 1995).

6. Both bulge and disk surface brightnesses correlate with respective scale lengths, consistently with the projection of the fundamental plane for ellipticals and spiral bulges (e.g., Andredakis et al. 1995). We note that uncertainties in the decomposition, especially in the shape index $n$, strongly influence the position of a bulge within the FP. Disks appear to reside in a region of this FP projection which is roughly contiguous to that of bulges, extending the correlation to larger radii and fainter surface brightnesses.

7. We confirm the tendency for the ratio of bulge and disk scale lengths $r_{\mathrm{e}} / r_{\mathrm{d}}$ to be constant, noted by de Jong (1996a) and Courteau et al. (1996). However, we find a mean (best- $n$ ) value $r_{\mathrm{e}} / r_{\mathrm{d}}=0.3$, significantly larger than the value found by de Jong and Courteau et al.; our $n=1$ value of $r_{\mathrm{e}} / r_{\mathrm{d}}$ of 0.2 agrees roughly with their value of $0.13-0.14$, while our $n=4$ value is 0.7 , more than a factor of 3 larger. We attribute such differences to different bulge parameterizations and caution that if best $n$ varies with morphological type, as suggested by Andredakis et al. (1995), $r_{\mathrm{e}} / r_{\mathrm{d}}$ may not be constant with morphological type, and thus the Hubble sequence may not be scale free as proposed by Courteau et al. (1996).

Acknowledgements. We would like to thank the referee, F. Simien, for a thorough reading of the manuscript and useful suggestions. This research was partially funded by ASI Grant 95-RS-120.

\section{Appendix A}

\section{Generalized exponential brightness distributions and conversion factors}

Assuming circular symmetry, we define a generalized exponential bulge as a surface brightness distribution:

$I(r)=I_{0} \exp \left[-\left(\frac{r}{r_{0}}\right)^{1 / n}\right]$ where $r$ is the projected radius, $I_{0}$ the central brightness, $r_{0}$ the exponential folding length, and $n$ an integer index $(n \geq 1)$. With the change of variable $x^{n}=r / r_{0}$, it is easily seen that the total luminosity $L$ is:

$L=\int_{0}^{\infty} I(r) 2 \pi r \mathrm{~d} r=\pi(2 n) ! I_{0} r_{0}^{2}$.

It is customary to introduce instead of $r_{0}$ an effective radius $r_{\mathrm{e}}$, which encircles half of the luminosity, and instead of $I_{0}$ an effective brightness $I_{\mathrm{e}}=I\left(r_{\mathrm{e}}\right)$. We derive in the following the relations between these quantities in the case of a generalized exponential.

The relation defining $r_{\mathrm{e}}$ is:

$\int_{0}^{r_{\mathrm{e}}} I(r) 2 \pi r \mathrm{~d} r=\frac{L}{2}$

with the above mentioned change of variable, this integral yields:

$\mathrm{e}^{-\alpha_{n}} \sum_{i=0}^{2 n-1} \frac{\alpha_{n}^{2 n-1-i}}{(2 n-1-i) !}=\frac{1}{2}$, with $\alpha_{n}=\left(\frac{r_{\mathrm{e}}}{r_{0}}\right)^{\frac{1}{n}}$

which we write as:

$\mathrm{e}^{-\alpha_{n}} \sum_{i=0}^{m} \frac{\alpha_{n}^{i}}{i !}=\frac{1}{2} \quad$ with $\quad m=2 n-1$.

The left hand side of this last equation is a cumulative Poisson distribution with parameter $\alpha_{n}$. This can be written in terms of $\chi^{2}$ probability functions (Abramowitz \& Stegun 1971):

$\mathrm{e}^{-\alpha_{n}} \sum_{i=0}^{m} \frac{\alpha_{n}^{i}}{i !}=Q\left(\chi^{2} \mid \nu\right)$

with $\chi^{2}=2 \alpha_{n}$ and $\nu=2(m+1)=4 n$.

$\alpha_{n}$ is therefore easily evaluated from $\chi^{2}$ tables. In any case, for large $\nu$ values, $\left(\chi^{2} / \nu\right)^{1 / 3}$ is approximately normally distributed:

$Q\left(\chi^{2} \mid \nu\right) \simeq Q\left(x_{2}\right)$

with $\quad x_{2}=\frac{\left(\frac{\chi^{2}}{\nu}\right)^{\frac{1}{3}}-\left(1-\frac{2}{9 \nu}\right)}{\sqrt{\frac{2}{9 \nu}}}$.

Since in our case $Q\left(x_{2}\right)=\frac{1}{2}$, it follows $x_{2}=0$ and $\chi^{2} \simeq$ $\nu\left(1-\frac{2}{3 \nu}\right)$. Then, with the approximations adopted:

$\alpha_{n} \simeq 2 n-\frac{1}{3}, \quad$ or $\quad r_{\mathrm{e}} \simeq r_{0}\left(2 n-\frac{1}{3}\right)^{n}$.

It turns out that the approximation is already quite good for $n=1$, with a relative error of $0.7 \%$. Table A1 reports the solution of Eq. (A5) and its approximation with Eq. (A9) for various values of $n$.

For the effective surface brightness we have:

$$
\begin{aligned}
I_{\mathrm{e}} & =I\left(r_{\mathrm{e}}\right)=I_{0} \exp \left[-\left(\frac{r_{\mathrm{e}}}{r_{0}}\right)^{\frac{1}{n}}\right] \\
& =I_{0} \mathrm{e}^{-\alpha_{n}} \simeq I_{0} \exp \left(-2 n+\frac{1}{3}\right) .
\end{aligned}
$$

These results are easily generalized to elliptical distributions and they are found to hold in the same form if $r_{\mathrm{e}}$ is the major semiaxis of the ellipse encircling half luminosity. 
Table 5. $r_{\mathrm{e}}$ approximation

\begin{tabular}{rrrr}
\hline$n$ & $\nu$ & $\alpha_{n}$ & $2 n-\frac{1}{3}$ \\
\hline 1 & 4 & 1.6784 & 1.6667 \\
2 & 8 & 3.6721 & 3.6667 \\
3 & 12 & 5.6702 & 5.6667 \\
4 & 16 & 7.6693 & 7.6667 \\
6 & 24 & 11.6684 & 11.6667 \\
10 & 40 & 19.6677 & 19.6667 \\
\hline
\end{tabular}

\section{References}

Abramowitz M., Stegun I.A., 1971, Handbook of Mathematical Functions. Dover, New York

Andredakis Y.C., Peletier R.F., Balcells M., 1995, MNRAS 275,874

Bertola F., Cinzano P., Corsini E.M., et al., 1996, ApJ 458, L67

Bianchi S., 1995, "Laurea" thesis, University of Florence

Bianchi S., Ferrara A., Giovanardi C., 1996, ApJ 465, 127

Binggeli B., Sandage A., Tamman G.A., 1985, AJ 90, 1681

Boroson T., 1981, ApJS 46, 177

Byun Y.I., Freeman K.C., 1995, ApJ 448, 563

Byun Y.I., Freeman K.C., Kylafis N.D., 1994, ApJ 432, 114 (BFK)

Casali M.M., Hawarden T.G., 1992, JCMT-UKIRT Newsletter No. 4 , p. 33

Cardelli A.J., Clayton G.C., Mathis J.S., 1989, ApJ 345, 245

Courteau S., de Jong R.S., Broeils A.H., 1996, ApJ 457, L73

David L.P., Jones C., Forman W., 1992, ApJ 388, 82

de Jong R.S., 1996a, A\&A 313, 45

de Jong R.S., 1996b, A\&A 313, 377

de Vaucouleurs G., de Vaucouleurs A., Corwin H.G., et al., 1991, Third Reference Catalog of Bright Galaxies. Springer Verlag, New York (RC3)

Devereux N., 1987, ApJ 323, 91

Disney M., Davies J., Phillipps S., 1989, MNRAS 239, 939

Djorgovski S., Davis M., 1987, ApJ 313, 59

Eder J., Giovanelli R., Haynes M.P., 1991, AJ 102, 572

Evans R., 1994, MNRAS 266, 511

Fillmore J.A., Boroson T.A., Dressler A., 1986, ApJ 302, 208

Frogel J.A., 1985, ApJ 298, 528
Frogel J.A., Persson S.E., Aaronson M., Matthews K., 1978, ApJ 220, 75

Garcia A.M., 1993, A\&AS 100, 47

Giovanardi C., Hunt L.K., 1988, AJ 95, 408

Giovanardi C., Hunt L.K., 1996, AJ 111, 1086

Giovanelli R., Haynes M.P., Salzer J.J., et al., 1995, AJ 110, 1059

Geller M.J., Huchra J.P., 1983, ApJS 52, 61

Hjorth J., Madsen J., 1995, ApJ 445, 55

Hoessel J.G., Schneider D.P., 1985, AJ 90, 1648

Huchra J., Burg R., 1992, ApJ 393, 90

Huchra J.P., Geller M.J., 1982, ApJ 257, 423

Hunt L.K., Calamai G., Oliva E., 1987, Arcetri Technical Report $4 / 87$

Hunt L.K., Malkan M.A., Salvati M., et al., 1997, ApJS 108, 229

Kent S.M., 1985, ApJS 59, 115

Kent S.M., 1986, AJ 91, 1301

Kent S.M., 1988, AJ 96, 514

Kodaira K., Watanabe M., Okamura S., 1986, ApJS 62, 703

Kormendy J., 1977, ApJ 218, 333

Kormendy J., Djorgovsky S., 1989, ARA\&A 27, 235

Knapen J.H., van der Kruit P.C., 1991, A\&A 248, 57

Lisi F., Baffa C., Biliotti V., et al., 1996, PASP 108, 364

Mihalas D., Binney J., 1981, Galactic Astronomy. Freeman, San Francisco

Peletier R.F., Willner S.P., 1992, AJ 103, 1761

Peletier R.F., Valentijn E.A., Moorwood A.F.M., Freudling W.F., 1994, A\&AS 108, 621

Pogge R.W., Eskridge P.B., 1993, AJ 106, 1405

Prugniel Ph., Simien F., 1997, A\&A 321, 111

Rix H-W., Rieke M.J., 1993, ApJ 418, 123

Rubin V.C., Burstein D., Ford W.K., Thonnard N., 1985, ApJ 289, 81

Sage L., 1993, A\&AS 100, 537

Schombert J.M., Bothun G.D., 1987, AJ 92, 60

Sèrsic J.-L., 1982, Extragalactic Astronomy. Reidel, Dordrecht

Soifer B.T., Boehmer L., Neugebauer G., Sanders D.B., 1989, AJ 98, 766

Sparks W.B., 1988, AJ 95, 1569

Terndrup D.M., Davies R.L., Frogel J.A., DePoy D.L., Wells L.A., 1994, ApJ 432, 518

Young J.S., Xie S., Tacconi L., et al., 1995, ApJS 98, 219 

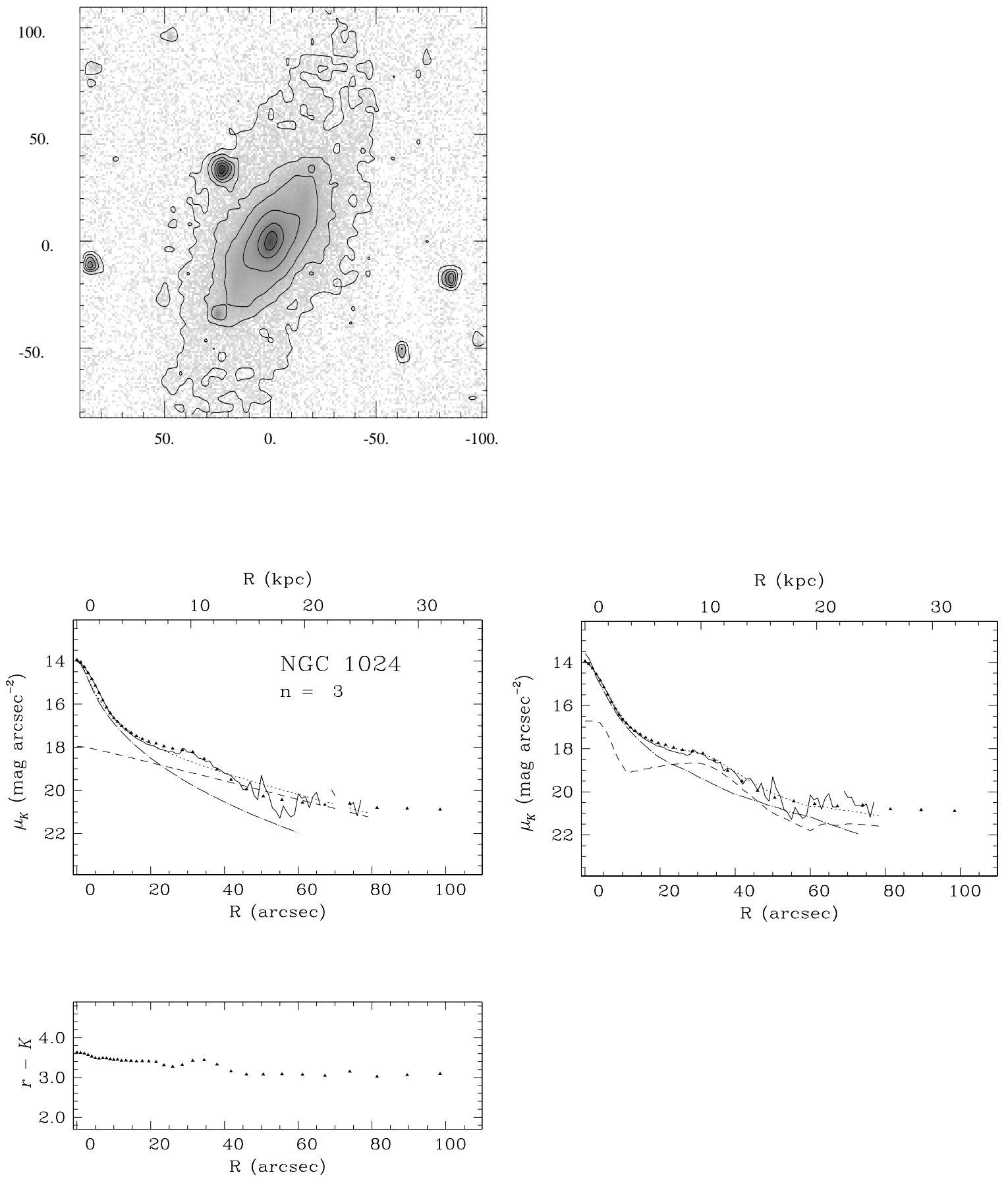

Fig. 1. $K$-band images, $J-K$ color images, parametric, and non-parametric decompositions. The upper left panel shows the $K$-band map; contours range from 21 to $14 \mathrm{mag} \operatorname{arcsec}^{-2}$ in unit steps. Images are oriented with North up and East left, and offsets are given in arcsec. The upper right panel shows the $J-K$ color map, with the color table ranging from 0.5 (black) to 1.3 (white). The lower left panels show the results of the parametric decomposition with the $K$-band elliptically-averaged radial profiles shown as filled triangles, and the major-axis cut shown as a solid line. The parametric bulge and disk are shown as dot-dashed and dashed lines, respectively, and their sum as a dotted line. 28" aperture profiles (Giovanardi \& Hunt 1996) are shown as large filled circles, if available. The remaining lower left panels show the elliptically-averaged profiles in $J-K$, and $r-K$ with the $r$-band taken from Kent (1988), if available. Also shown in the $J-K$ plots are the fitted color profile (dotted line), and the fitted colors of bulge (dot-dashed line) and disk (dashed line). The lower right panel shows the results of the non-parametric decompositions, with the same coding as for the parametric decompositions 

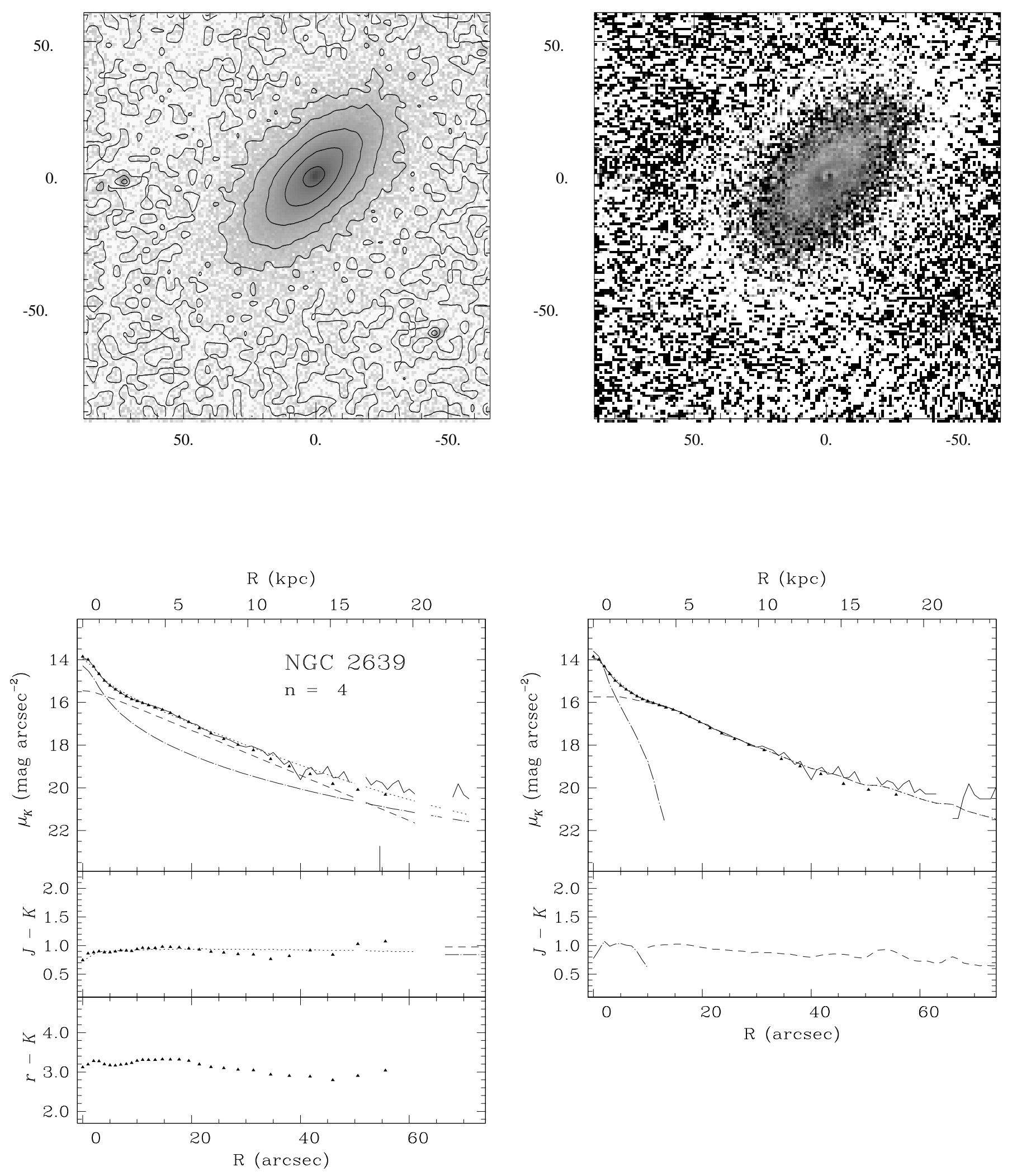

Fig. 1. continued 

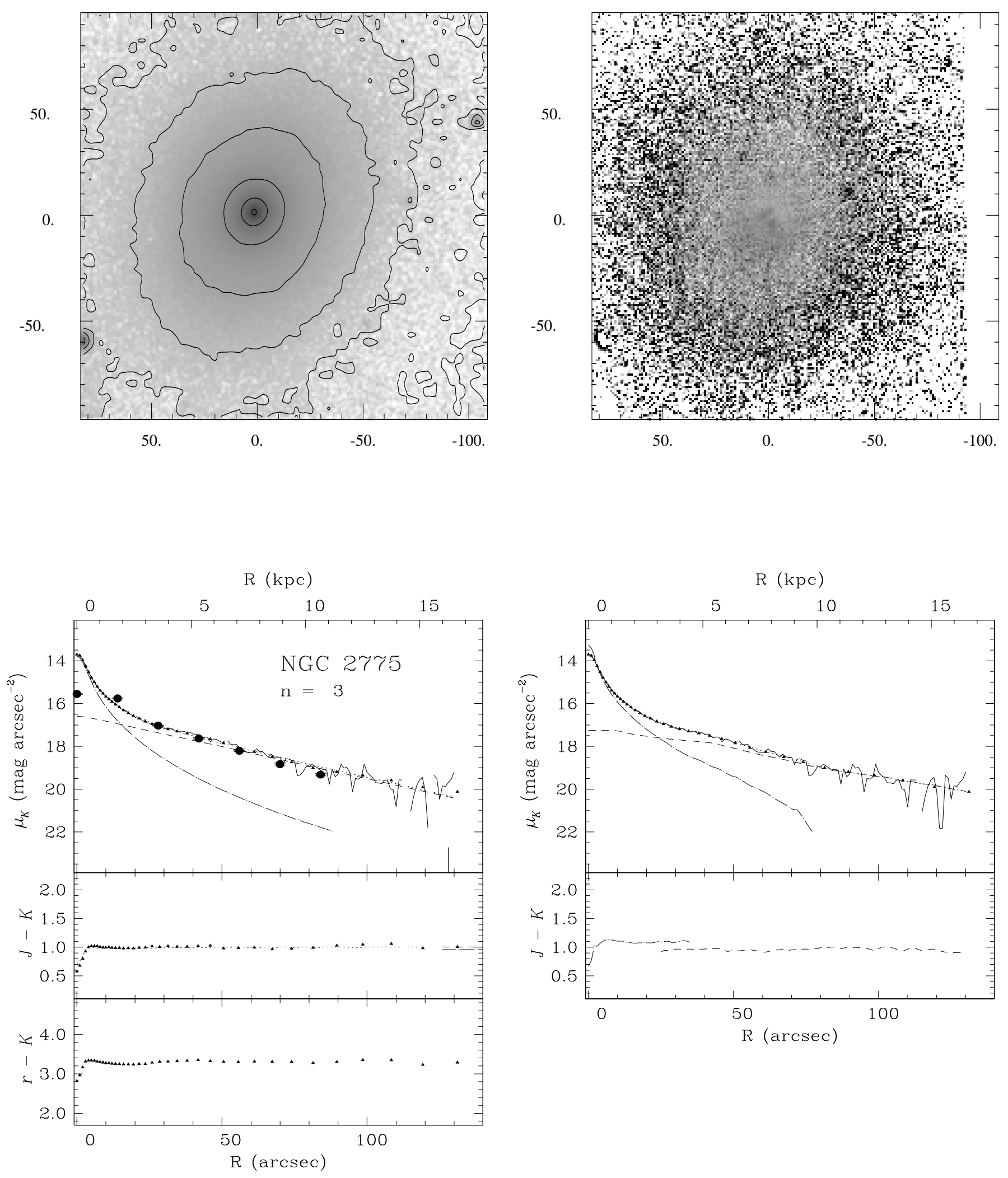

Fig. 1. continued 

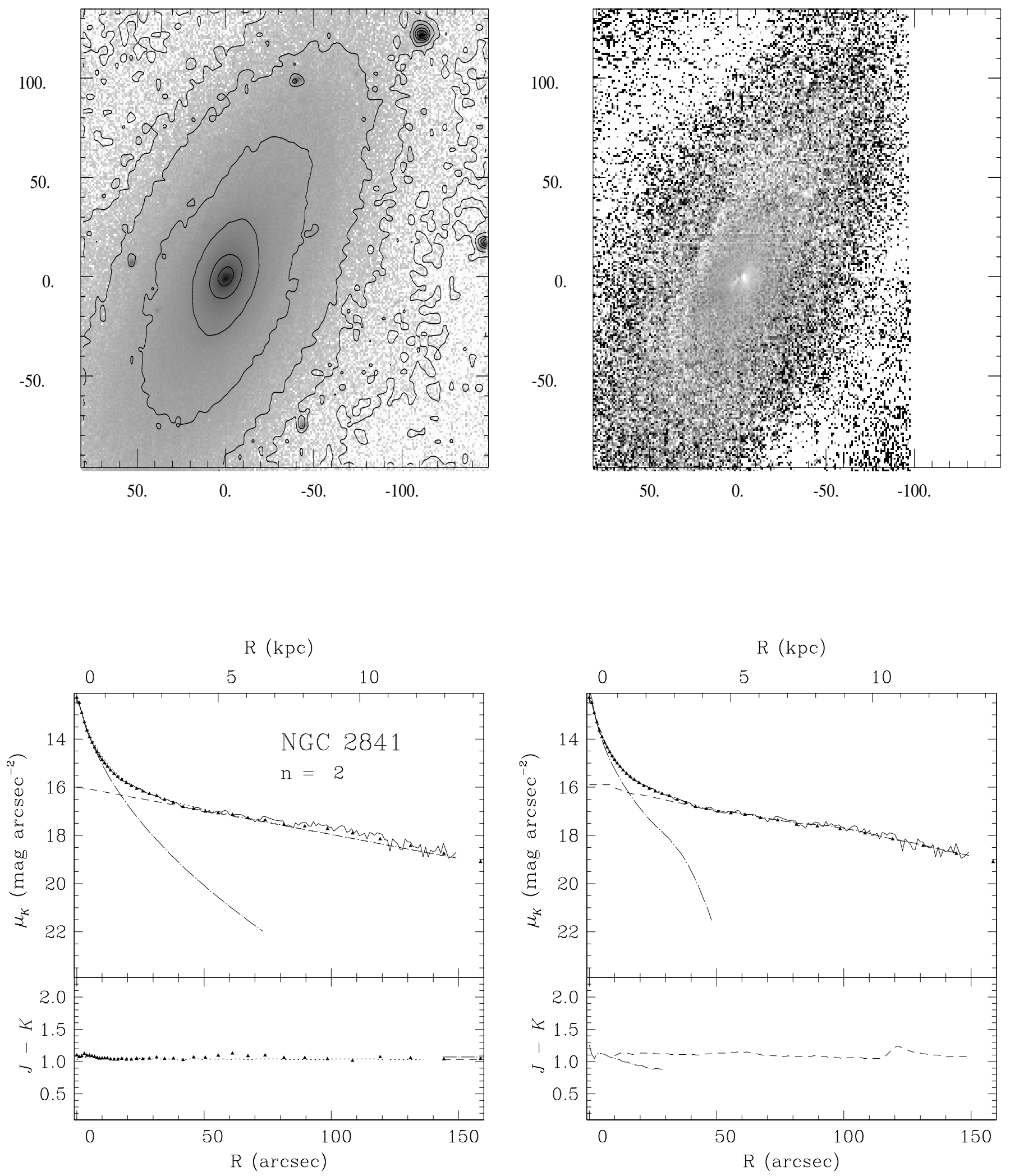

Fig. 1. continued 

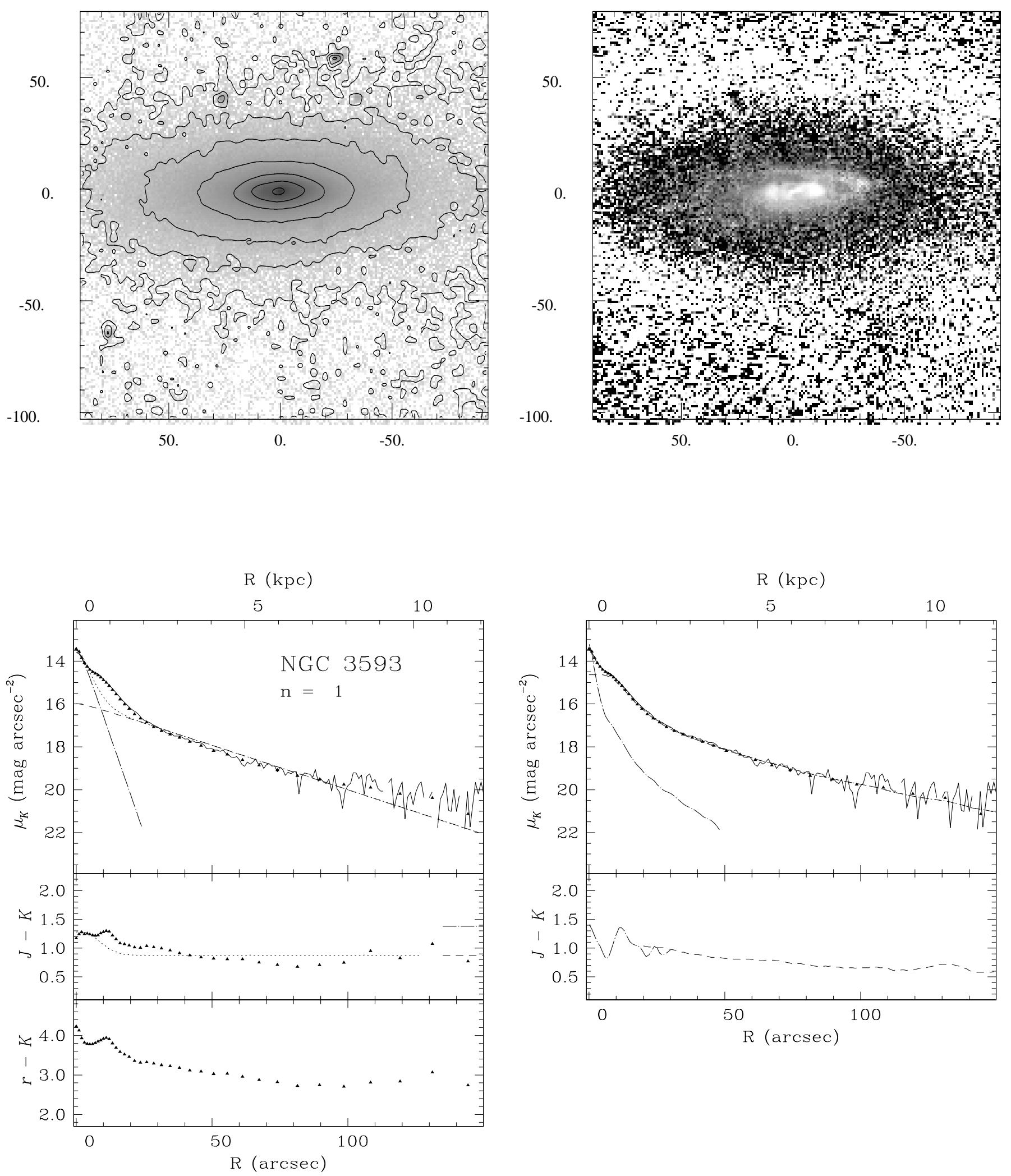

Fig. 1. continued 

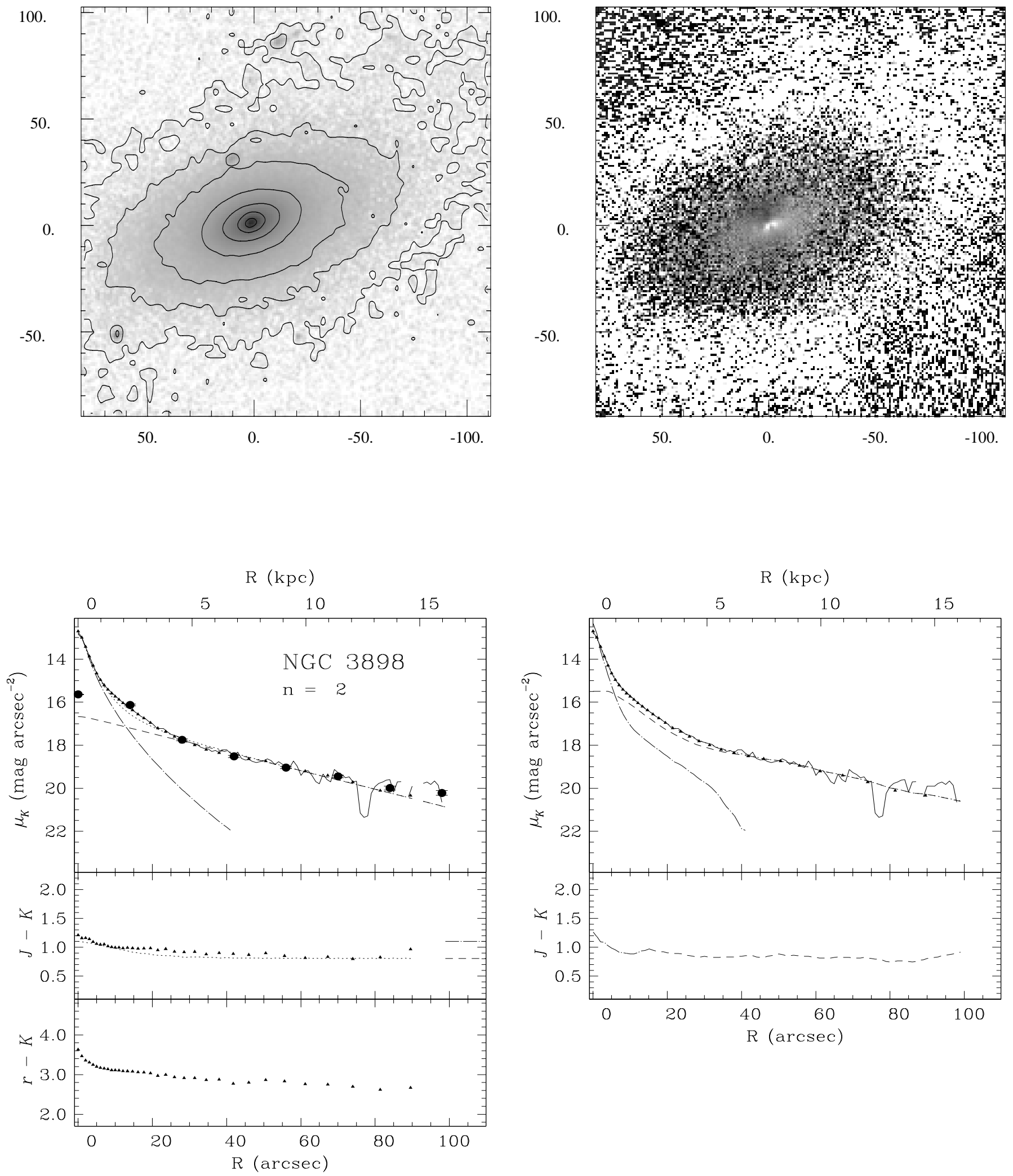

Fig. 1. continued 

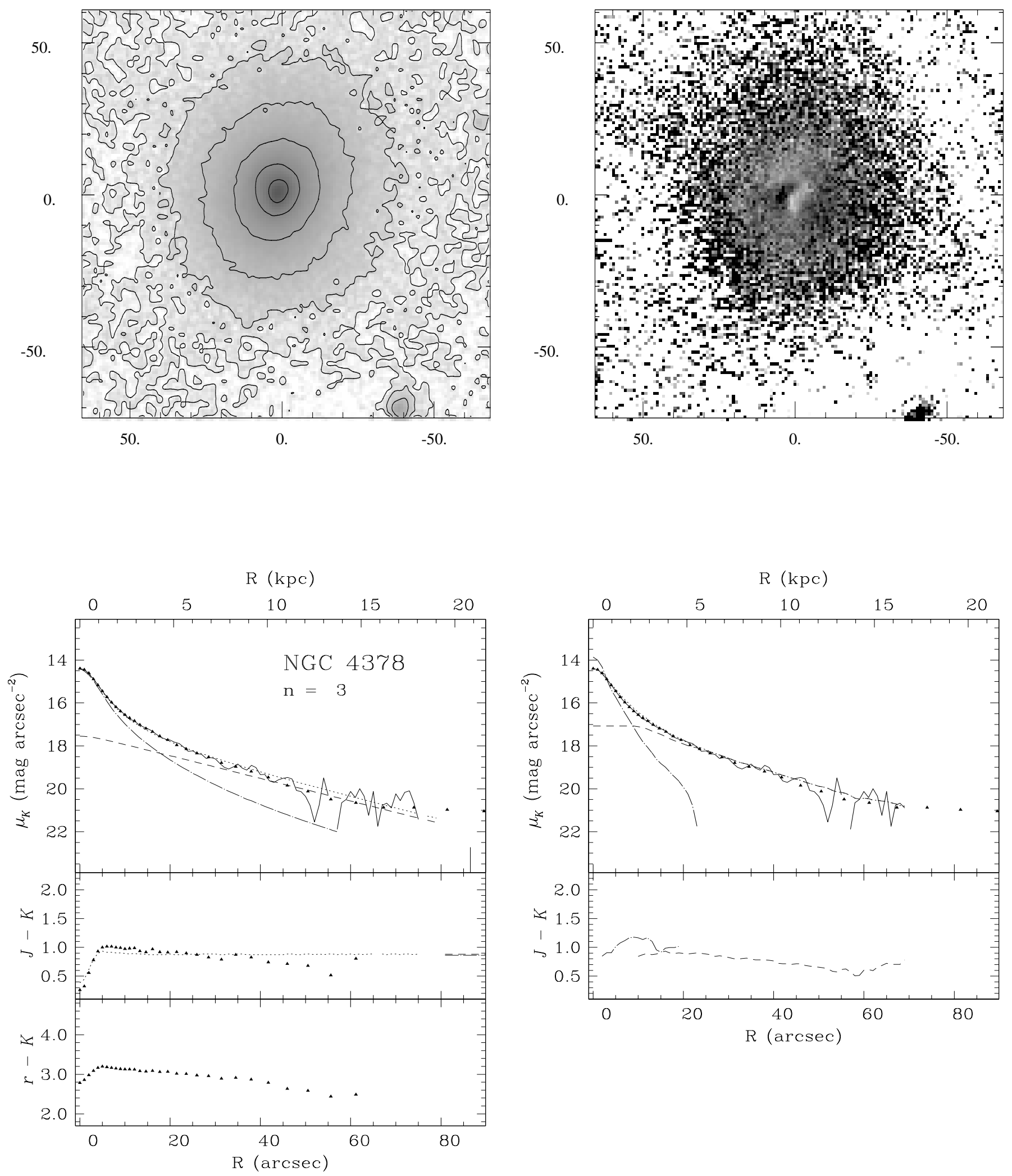

Fig. 1. continued 

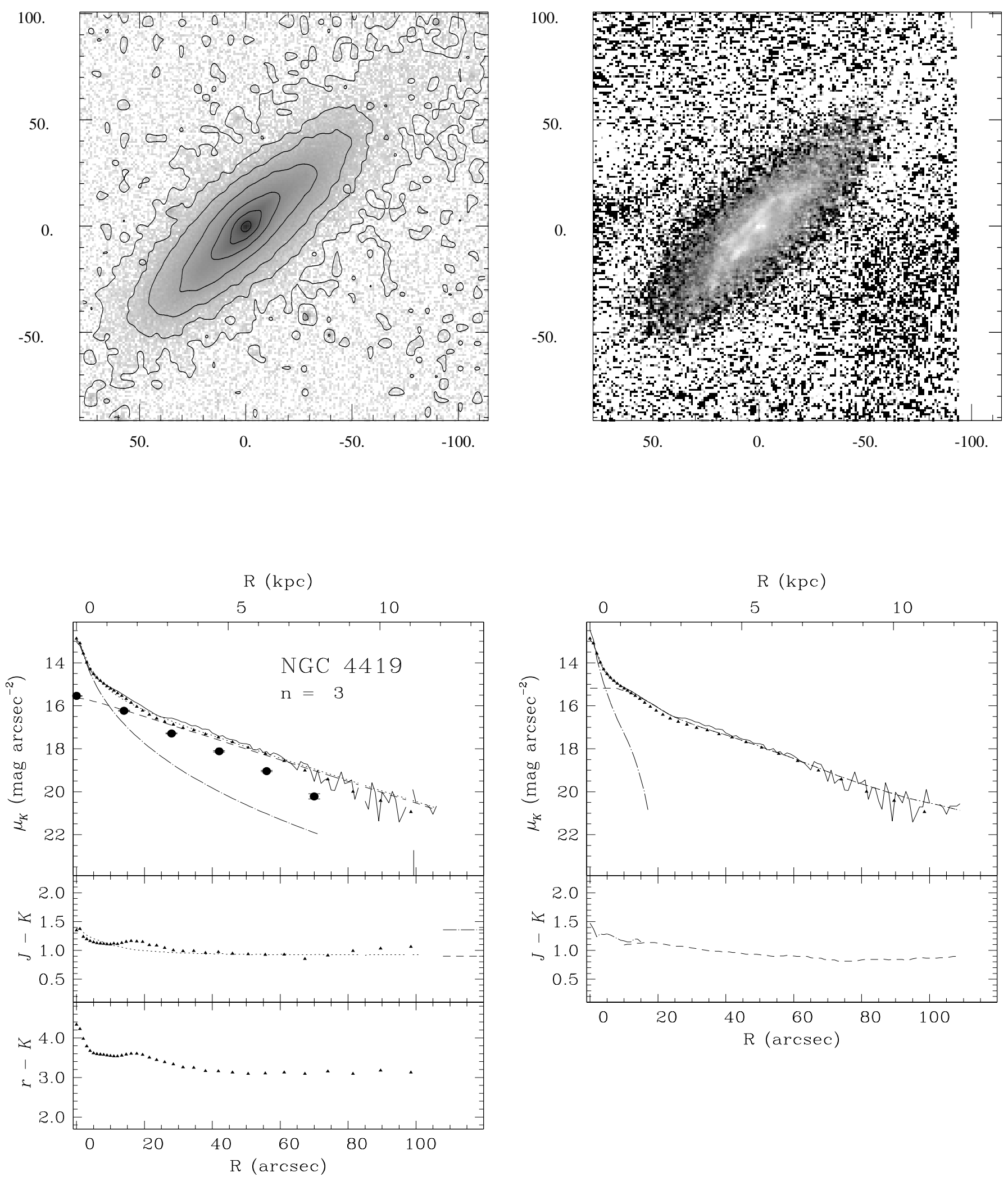

Fig. 1. continued 

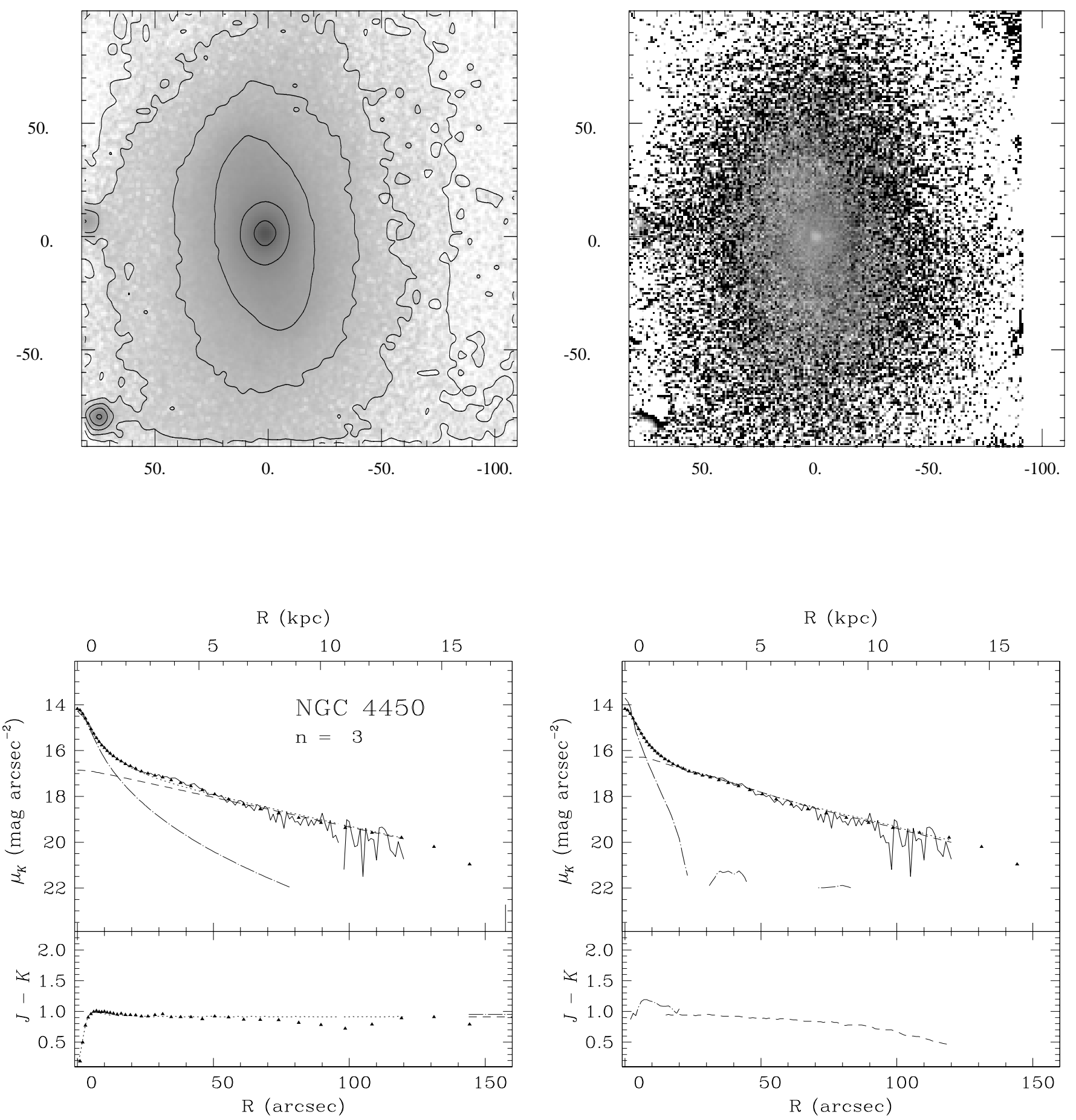

Fig. 1. continued 

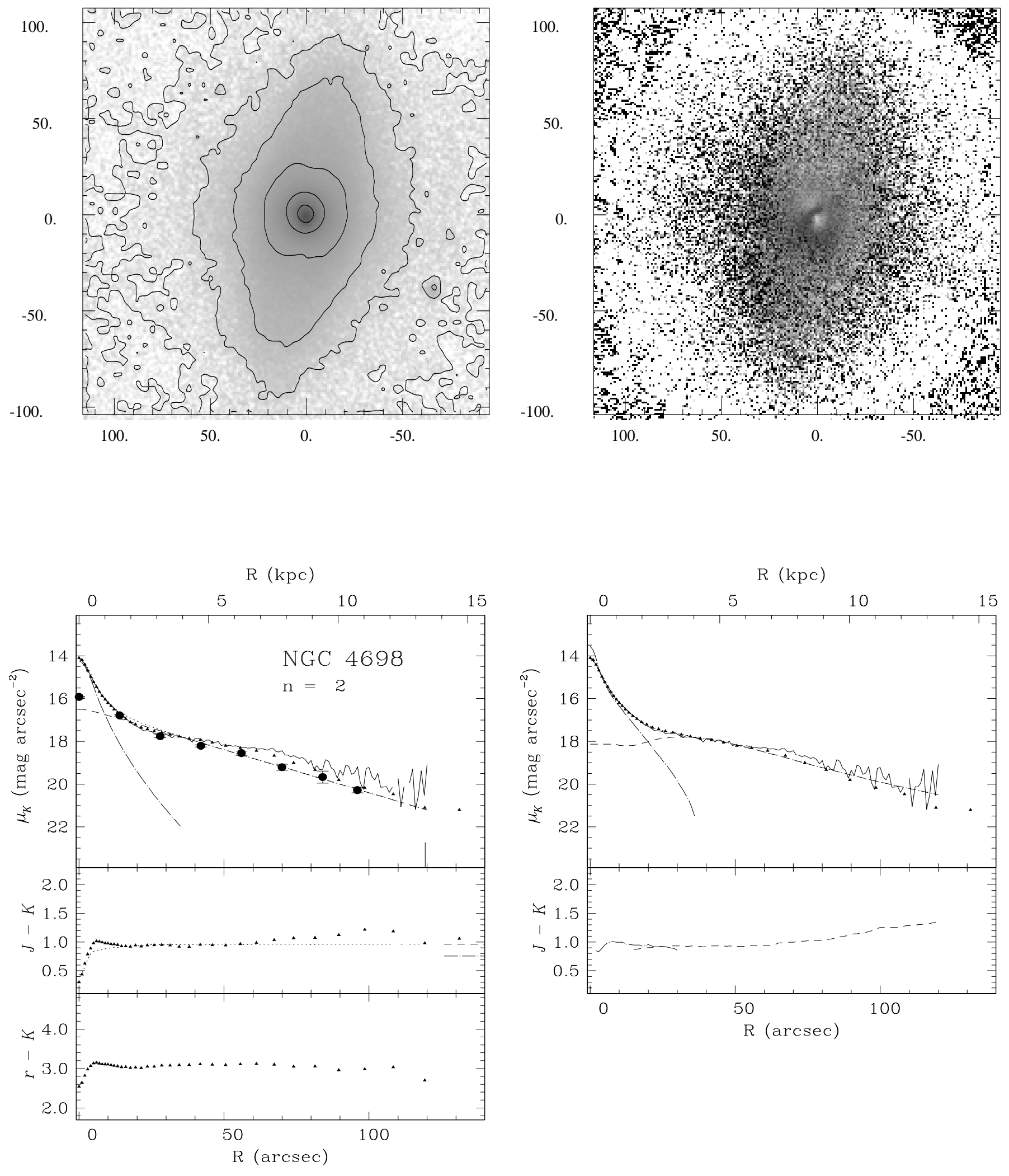

Fig. 1. continued 

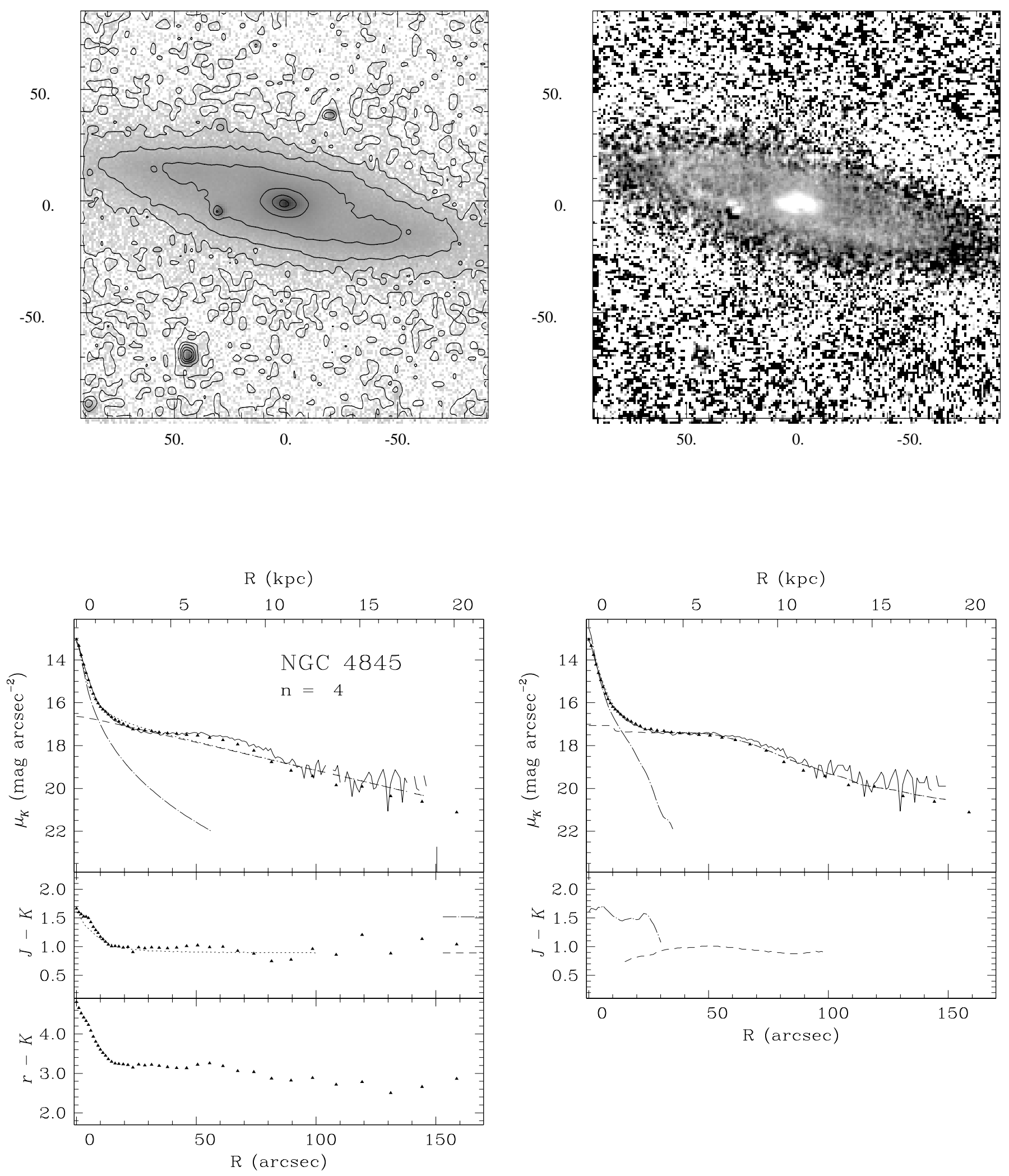

Fig. 1. continued 

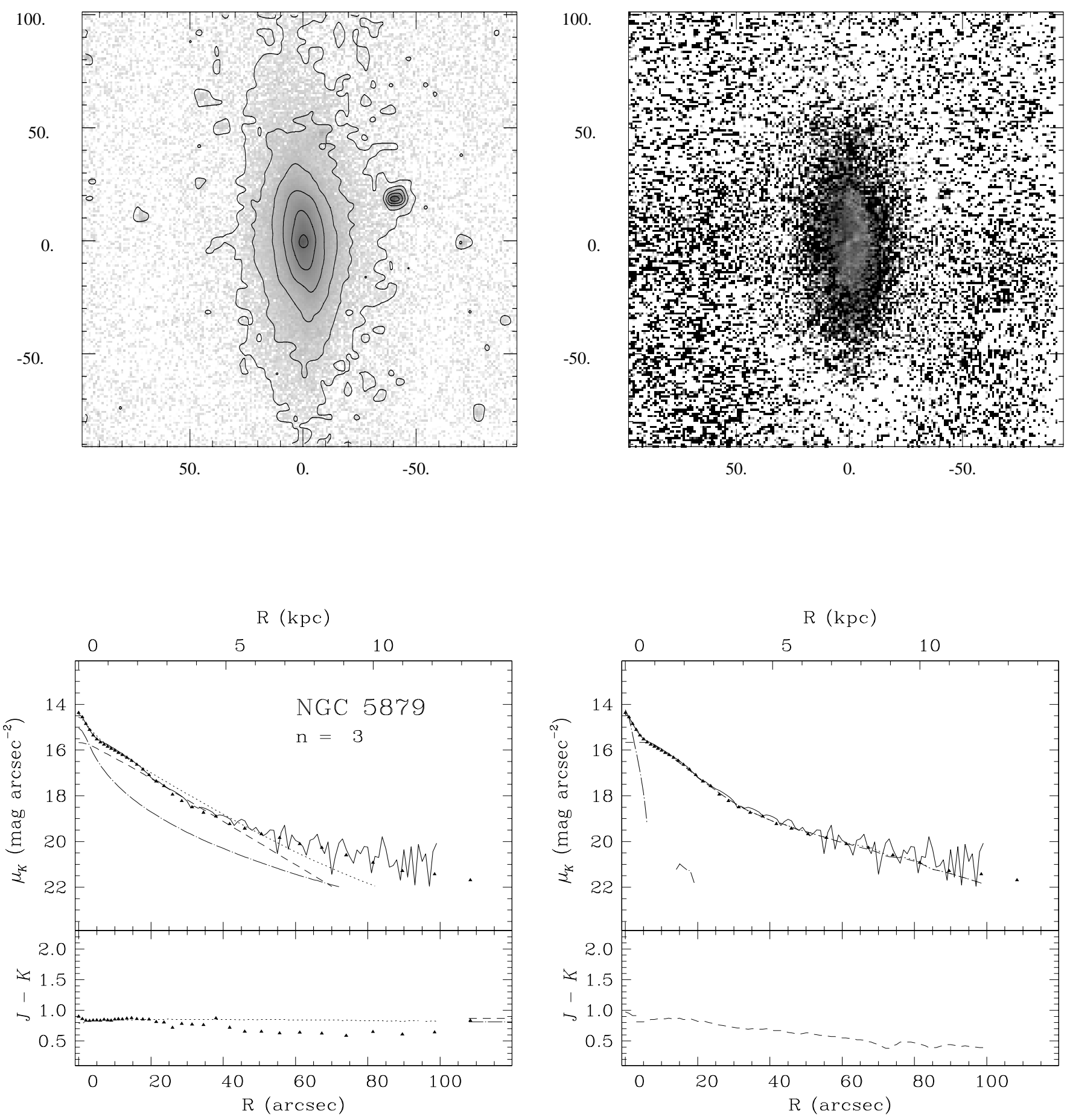

Fig. 1. continued 

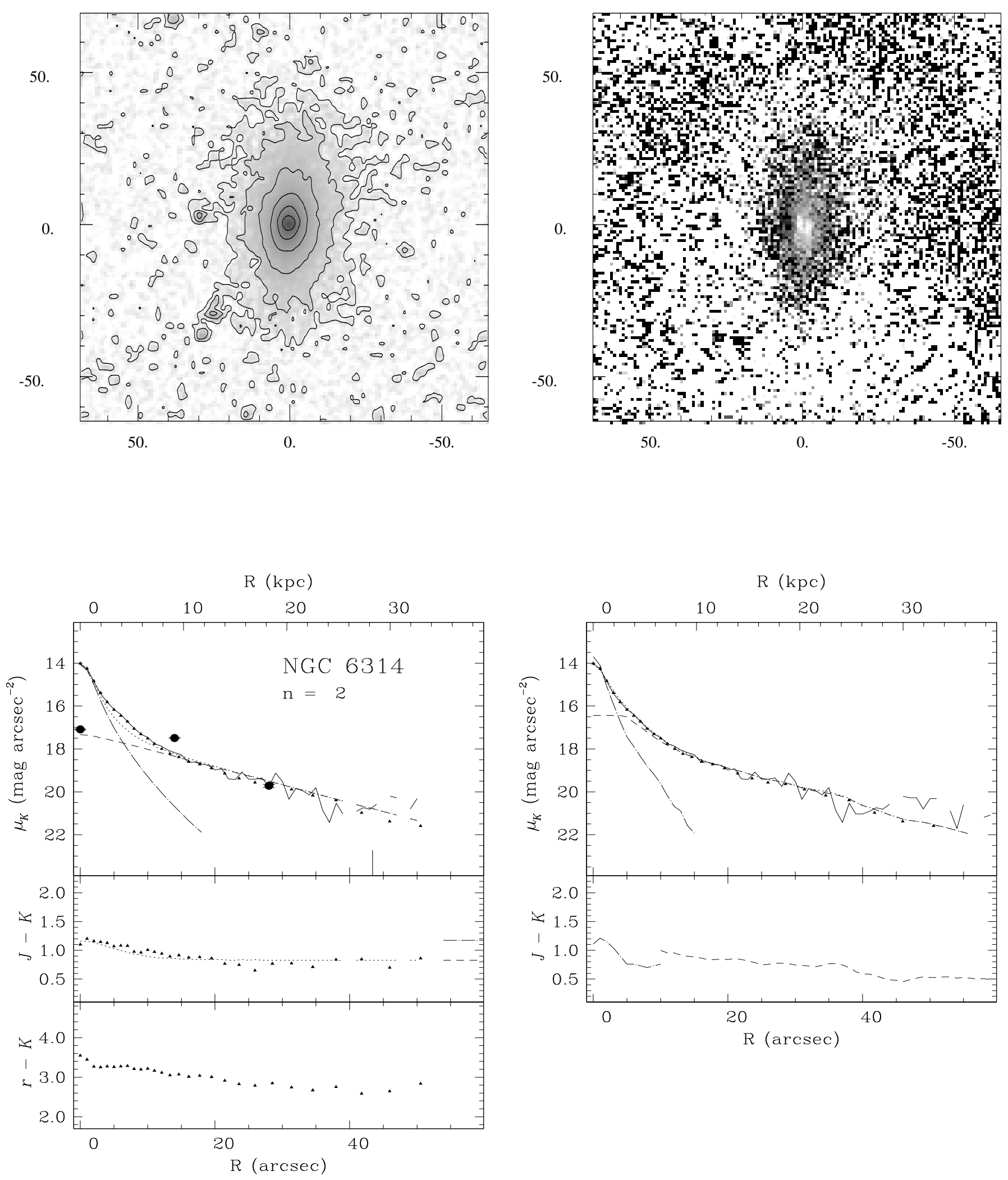

Fig. 1. continued 

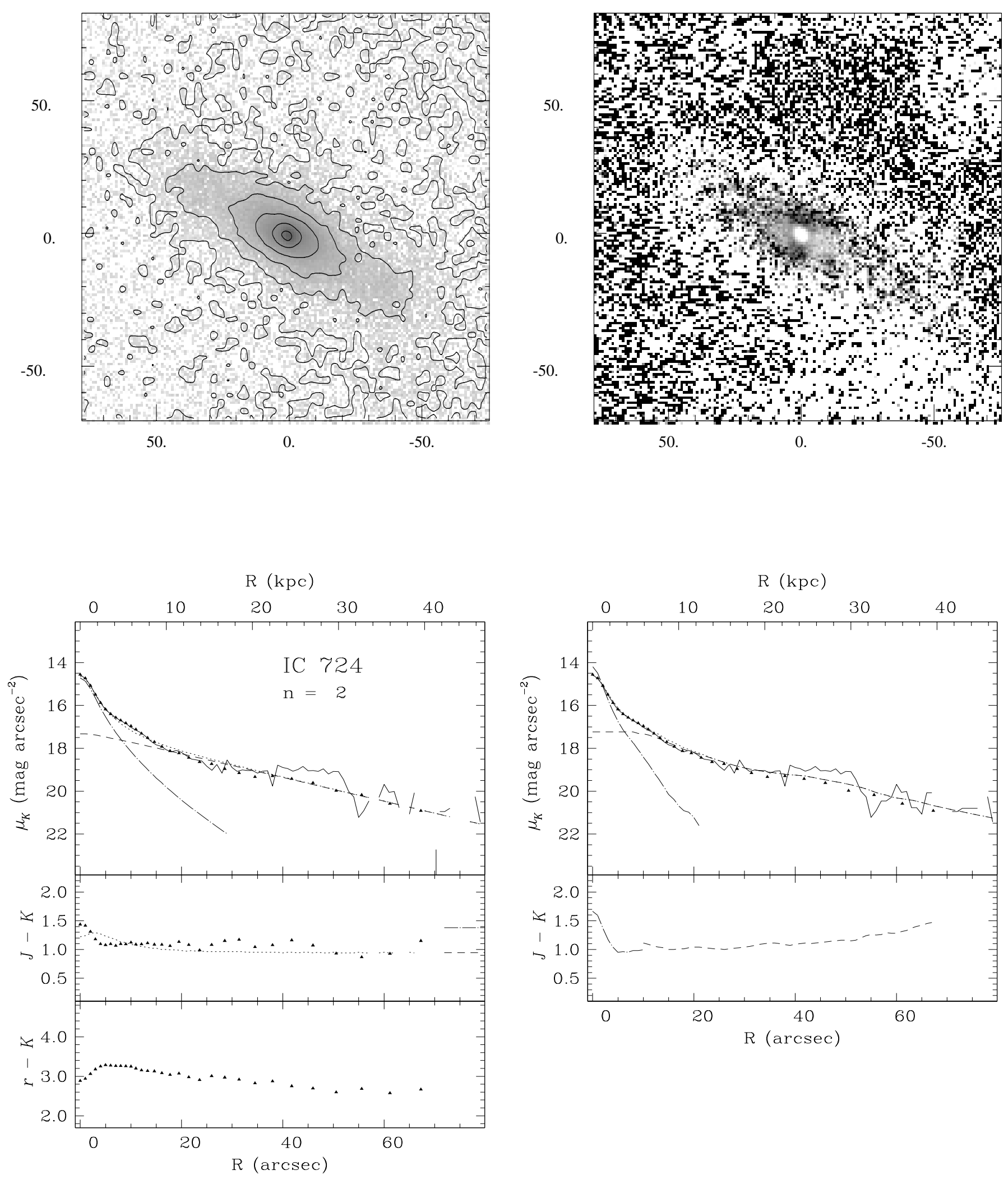

Fig. 1. continued 